\title{
Understanding the CO Pre-Oxidation and the Intrinsic Catalytic Activity of Step Sites in Stepped Pt Surfaces in Acidic Medium
}

\author{
Manuel J.S. Farias ${ }^{1, *}$, Giuseppe A. Camara ${ }^{1}$, Juan M. Feliu ${ }^{2}$ \\ ${ }^{1}$ Instituto de Química, Universidade Federal de Mato Grosso do Sul, C.P. 549, 79070- \\ 900, Campo Grande, Brazil \\ ${ }^{2}$ Instituto de Electroquímica, Universidad de Alicante Ap. 99, E-03080, Alicante, Spain
}

\begin{abstract}
In order to deepen the knowledge about the origin of the CO pre-oxidation process and the intrinsic catalytic activity of Pt superficial steps toward $\mathrm{CO}$ oxidation were performed a series of CO stripping experiments on stepped Pt electrodes in acidic medium. For the occurrence of $\mathrm{CO}$ pre-oxidation, it was found that it arises (reproducibly) whenever four interconnected conditions are simultaneously fulfilled: (1) $\mathrm{CO}$ adsorption at potentials lower than about $0.2 \mathrm{~V}$; (2) on surfaces saturated with $\mathrm{CO}_{\text {ads }}$; (3) in presence of traces of $\mathrm{CO}$ in solution; (4) in presence of surface steps. If any of these four conditions is not satisfied, the CO pre-oxidation pathway does not appear, even though the steps on the electrode surface are completely covered by CO. By controlling the removal of the CO adlayer (voltammetrically), we show that once the CO adlayer has been partially oxidized, the (111) terrace sites of stepped surfaces are released earlier than the (110) step sites. Moreover, if (110) steps are selectively decorated with CO, its oxidation occurs only at potentials $\sim 150 \mathrm{mV}$ higher than the CO pre-oxidation peak. Our results systematically demonstrate that step sites are less active to oxidize CO than those ones responsible for the CO pre-oxidation process. Once the sites responsible for the $\mathrm{CO}$ pre-oxidation are made free, there is no apparent motion of the remaining adsorbed CO layer, suggesting that the activation of the surface controls
\end{abstract}


the whole process, rather than the diffusion of $\mathrm{CO}_{\mathrm{ads}}$ towards hypothetically "most active sites”. Voltammetric and chronoamperometric experiments performed on partially covered CO adlayers suggest that adsorbed CO behave as a motionless species during its oxidation, in which the $\mathrm{CO}$ adlayer is removed piece by piece. By means in situ FTIR experiments the stretching frequency of CO selectively adsorbed on (110) step sites was examined. Band frequency results confirm that those molecules adsorbed on steps are fully coupled with the adsorbed CO on (111) terraces when the surface reaches full coverage.

Keywords: Electrocatalysis, CO pre-oxidation, stepped Pt electrodes, catalytic activity of step sites, CO surface diffusion.

*Corresponding Author:

manueljsfarias@gmail.com (M.J.S. Farias). Phone: +55 6733453596 


\section{Introduction}

It is well known that the oxidative stripping of $\mathrm{CO}$ from a pure Pt electrode requires high over-potentials. The standard electrode potential for the reaction $\mathrm{CO}_{(g)}+$ $\mathrm{H}_{2} \mathrm{O}_{(l)} \rightleftarrows \mathrm{CO}_{2(g)}+2 \mathrm{H}^{+}+2 e^{-}$is $E^{0} \simeq-0.103$ vs. SHE/V, but experimentally on pure Pt electrodes, the CO electro-oxidation reaction starts only at about 0.7 vs. RHE/V at $\sim 25{ }^{\circ} \mathrm{C}$ (room temperature). Nonetheless, under some experimental conditions, certain specific reaction pathways for CO oxidation are activated at lower potentials, through which the CO adlayer might be partially oxidized. Models to explain the CO preoxidation process - as they are often named nowadays - are a topic heavily debated in literature. To the best of our knowledge, the CO pre-oxidation process was firstly reported by Grambow and Bruckenstein ${ }^{1}$ and about 10 years later was again reported by Kita et al.. ${ }^{2}$ The later authors found that the pre-oxidation took place when CO was adsorbed in the hydrogen potential deposition $\left(\mathrm{H}_{\text {upd }}\right)$ region, which lead them to conclude that the pre-oxidation of CO was linked to its adsorption potential ( $\left.E_{\text {ads }}\right)$.

From the point of view of electrocatalysis, the pre-oxidation pathway is a major subject concerning the electro-oxidation of $\mathrm{CO}$ [with special interest in $\mathrm{Pt}(h k l) / \mathrm{CO}$ system], since it anticipates the oxidation of this molecule, that is a poison for hydrogen fuel cells and also is at the heart of the electro-oxidation of alcohols and related species. $^{3-4}$ Despite its importance, in voltammetric experiments of CO stripping the strict control of the conditions to promote this pre-oxidation is not straightforward, and there is a lack of consensus in that subject in different laboratories worldwide. In other words, several experiments under "similar" conditions (i.e. same potential of adsorption of CO, same supporting electrolyte, similar conditioning of the electrode surface and of their crystallographic orientations), show the CO pre-oxidation, ${ }^{5-7}$ while another results never show it. ${ }^{8-9}$ Moreover, the pre-oxidation of CO has not yet been observed for CO 
stripping voltammetry even on some Pt(111) vicinal surfaces in acidic medium. ${ }^{10-11}$ In light of its electrocatalytic importance, many hypotheses have been proposed to explain the origin of the CO pre-oxidation process, whether employing Pt polycrystalline ${ }^{12-14}$ or Pt single crystal ${ }^{5,9,15-19}$ electrodes in acidic media.

Under ultra-high vacuum conditions, Ertl et $a .^{20}$ showed that the heat of adsorption of CO decreases when its coverage increases. At high CO coverage, it seems that the heat of adsorption changes mainly due to lateral repulsive interactions (and to a lesser extent due to the occupation of different sites as terraces, steps and kinks). Marković et al. ${ }^{5,}{ }^{16}$ extrapolated the results of Ertl et $a .^{20}$ to electrochemical environments, and proposed that the CO pre-oxidation occurs due to the oxidation of weakly adsorbed $\mathrm{CO}$ present when the electrode surface is fully covered by $\mathrm{CO}$ ( $\theta_{\mathrm{CO}}$, max). According to this model, any CO molecule could be oxidized on any particular site during the CO pre-oxidation process, no matter whether they are on terrace sites or low coordinated ones. The core of this idea appeared before in a paper of Leiva et al.. ${ }^{21}$ On the other hand, López-Cudero et al. ${ }^{9,12,15}$ suggested that CO pre-oxidation is due to the CO oxidation by oxygenated species on step sites (defects). Steps on surface are considered the most active ones for the electro-oxidation of $\mathrm{CO}$, while terraces are considered poorly active sites for such reaction. ${ }^{22-24}$

Indeed, it has been found that the catalytic activity of Pt stepped electrodes towards the oxidation of $\mathrm{CO}$ increases with the step density in the series $\operatorname{Pt}(\mathrm{s})[(n-$ 1)(111) $\times(110)],{ }^{22}$ so that the extrapolation of the oxidation rate to a surface with a zero (110) step density [i.e., a perfect $\operatorname{Pt}(111)$ crystal in this zone] nicely coincides to the zero rate reaction. ${ }^{22}$ Consequently, it was assumed that CO on terraces quickly diffuses to steps, in which it exclusively oxidizes, ${ }^{22}$ following a Langmuir-Hinshelwood (L-H) type mechanism. ${ }^{25}$ 
According to these interpretations, in acid solution (in absence of anion adsorption) we would write the CO electro-oxidation reaction as the following:

$$
\begin{aligned}
& \mathrm{H}_{2} \mathrm{O}+\gamma \mathrm{Pt}_{\text {step }} \rightleftarrows \gamma \mathrm{Pt}_{\text {step }}-(\mathrm{OH})+\mathrm{H}^{+}+e^{-} \\
& v \mathrm{Pt}_{\text {step }}-\mathrm{CO}+\gamma \mathrm{Pt}_{\text {step }}-(\mathrm{OH}) \rightarrow \mathrm{CO}_{2}+(\gamma+v) \mathrm{Pt}_{\text {step }}+\mathrm{H}^{+}+e^{-}
\end{aligned}
$$

in which, like in the $\mathrm{CO}$ adsorption process, $\mathrm{CO}$ molecules adsorb on both step and terrace sites. Assuming that the reaction takes place exclusively on step sites, ${ }^{23-24}$ a high CO surface diffusion from terraces to steps has been claimed in equation (2), which should occur at low potentials (for instance, during CO pre-oxidation), whilst for CO adsorbed on terraces, its oxidation should take place only at higher electrode potentials. $^{22-24,26-27}$

However, it is not clear if during CO electro-oxidation, either it diffuses from any sites to steps or if such sites act promoting the activation of oxygen-containing species to oxidize CO at low potentials. Indeed, it is not even clear if steps really act as the most catalytic active sites on the surface for the CO electro-oxidation reaction. Regarding this topic it was recently suggested that it may be due to the oxidation of dissolved $\mathrm{CO}$ in specific sites available on a Pt (100)-(1×1) surface. ${ }^{28}$ A set of other conceptions about the origin of CO pre-oxidation was presented in a paper by Yan et al.. ${ }^{14}$ We therefore, reinvestigate these questions employing the $\mathrm{Pt}(h k l) / \mathrm{CO}$ system.

An overview of the literature reveals that the discussion concerning the catalytic activity of different superficial sites is based on CO stripping voltammetry and chronoamperometry. Namely, the Pt surface is fully covered by CO, whereafter the CO monolayer is oxidized at once. Under these conditions a unique CO oxidation wave is usually observed in acidic media, ${ }^{10,29}$ although the pre-oxidation wave is perceived in some cases. The narrow potential range in which the main CO oxidation peak takes place $(\Delta E \simeq 25 \mathrm{mV})$ means that eventual signals due to distinct crystalline planes 
emerge in a single oxidation peak. This means that responses coming from specific step sites remain inaccessible. Another attempt to access the catalytic activity of step sites was made by employing stepped surfaces containing terraces with different lengths, ${ }^{10}$ but even in this case a single CO oxidation peak is observed. The benchmarking of the catalytic activity of step sites towards CO oxidation in stepped Pt surfaces can be achieved provided that $\mathrm{CO}$ is selectively adsorbed on step sites, followed by its oxidation. By using this experimental strategy, the influence of CO adsorbed on terraces in the features of the stripping can be completely eliminated, and the resulting hydrogen region can be used as a diagnostic of the site occupancy and release. ${ }^{30}$

As the CO pre-oxidation process apparently depends on many parameters, it is paramount to control the topography of the surface on which the reaction takes place, so here we use well-characterized single crystal surfaces to approach questions concerning the issues raised above, i.e., the CO pre-oxidation, the role of step sites and the dynamics of adsorbed CO during its oxidation. Herein we investigate how experimental conditions, as the adsorption potential of $\mathrm{CO}$, the presence of steps on surface, the $\mathrm{CO}$ coverage and the presence of $\mathrm{CO}$ in solution influence the oxidation of $\mathrm{CO}$ at low potentials. In addition, we selectively decorate (110) step sites with CO and evaluate its intrinsic catalytic activity. Based on these results we are able to estimate the catalytic activity of step sites without the influence of CO coming from terraces, which we named "intrinsic catalytic activity" of step sites. In addition, by using in situ FTIR we were able to evaluate the stretching frequency of CO adsorbed on step sites without the interference caused by dipole-dipole coupling frequency (consequence of CO adsorbed on terraces).

\section{Experimental}


In this study, platinum single crystals with orientations $\operatorname{Pt}(111), \operatorname{Pt}(13,13,12)$, $\operatorname{Pt}(776)$ and $\operatorname{Pt}(554)$ (with areas between $\sim 3$ and $\sim 5 \mathrm{~mm}^{2}$ ), prepared according to the procedure described by Clavilier et al., ${ }^{31}$ have been used as working electrodes. The stepped surfaces mentioned above contain, respectively, 25, 13 and 9 atoms-wide (111) terraces separated by monoatomic steps with (110) symmetry. Models of hard spheres for these stepped surfaces are attached to Fig.1. According to Lang-Joyner-Somorjai, ${ }^{32}$ if we consider $n$ atoms-wide (111) terraces and (110) monoatomic steps, those vicinal stepped surfaces can be denoted as $\operatorname{Pt}(\mathrm{s})[(n-1)(111) \times(110)]$ or individually as:

$$
\begin{gathered}
\operatorname{Pt}(\mathrm{s})[25(111) \times(110)] \equiv \operatorname{Pt}(13,13,12) \\
\operatorname{Pt}(\mathrm{s})[13(111) \times(110)] \equiv \operatorname{Pt}(776) \\
\operatorname{Pt}(\mathrm{s})[9(111) \times(110)] \equiv \operatorname{Pt}(554)
\end{gathered}
$$

After flame annealing, the single crystals were cooled in a controlled $\mathrm{H}_{2} / \mathrm{Ar}$ atmosphere. Next, they were protected by a droplet of deoxygenated $\left(\mathrm{H}_{2} / \mathrm{Ar}\right)$ water and rapidly transferred to the electrochemical cell. A platinized platinum wire was used as a counter electrode and the reference electrode was a reversible hydrogen electrode (RHE), being all the potentials given in this scale. A $0.1 \mathrm{M} \mathrm{HClO}_{4}$ (Aldrich $^{\circledR} 70 \%$ ) solution prepared in ultrapure water (Milli-Q 18.2 M $\Omega \mathrm{cm}$ ) was used as electrolyte. $\mathrm{Ar}$ (Alpha Gaz ${ }^{\mathrm{TM}}$, N50) was used to deoxygenate the cell and the electrolytic solution. Afterwards, several voltammetric cycles were recorded with a Pt(111) surface in order to control the solution cleanness, and then the voltammetries of each stepped Pt electrode were recorded. Next, CO (Alpha Gaz ${ }^{\mathrm{TM}}$, N47) was bubbled into the solution for 5 min while the electrode potential was kept at $0.10 \mathrm{~V}$ (depending on the experiment). Finally, in order to remove excess of dissolved CO, the solution was purged with $\operatorname{Ar}$ ( $\sim 150 \mathrm{~mL} / \mathrm{min}$ in all experiments) for a controlled time ranging 5-60 
min. Every time an electrode surface was prepared (flame annealed), it was carried out a single CO stripping on that surface, so the CO stripping experiments were always performed on a fresh surface.

In situ Fourier Transform Infrared (FTIR) spectra were recorded employing a Nicolet (Model 8700) spectrometer, equipped with a Mercury-Cadmium-Telluride detector and cooled down with liquid $\mathrm{N}_{2}$. The spectroelectrochemical cell was fixed to a $60^{\circ}$ prismatic $\mathrm{CaF}_{2}$ window in a thin layer configuration. ${ }^{33} \mathrm{~A}$ p-polarized radiation was employed. According to the surface selection rule, ${ }^{34-35}$ it allows to detect active species both at the electrode surface and in the thin layer. ${ }^{33}$ The experiments consisted of collecting spectra (from the average of 100 interferograms with $8 \mathrm{~cm}^{-1}$ of resolution the acquisition time of each spectrum was about 55 s) while the electrode potential was stepped from $0.05 \mathrm{~V}$ to $0.70 \mathrm{~V}$, at intervals of $50 \mathrm{mV}$. Afterwards, the electrode potential was stepped to $0.900 \mathrm{~V}$ for collecting the reference spectrum. The spectra were collected and represented as absorbance $\left[A=-\log \left(R_{i} / R_{0}\right)\right.$ ], in which $R_{0}$ is a singlebeam reflectance reference spectrum (collected at $0.90 \mathrm{~V}$ ) and $R_{i}$ is a single beam reflectance spectrum at a sample potential. In this notation, positive bands appear for species formed into the thin layer, and negative ones when species are consumed or diffuse out thin layer.

All the experiments were performed at room temperature $\left(25^{\circ} \mathrm{C}\right)$. The electrode potentials for electrochemical and spectro-electrochemical experiments were controlled using a waveform generator (EG\&G PARC 175) together with a potentiostat (Amel 551) and a digital recorder (eDAC ED 401).

\section{Results}

\subsection{Electrochemical characterization of Pt single crystals}


Fig. 1 displays cyclic voltammograms of $\mathrm{Pt}(111), \operatorname{Pt}(13,13,12), \operatorname{Pt}(776)$ and Pt(554) electrodes. This figure also shows an illustration of a hard sphere model for each crystalline surface. For these surfaces, the step density $\rho_{(h k l)}$ (defined as the number of monoatomic steps per unit length in the plane of the terrace domain) follows the order: $\rho_{(554)}>\rho_{(776)}>\rho_{(13,13,12)}>>\rho_{(111)}$. For all surfaces, the voltammetric features and the stability of the voltammograms imply a high quality of the crystal surfaces and a solution free of impurities, respectively. Especially for $\operatorname{Pt}(111)$, both the magnitude of the reversible states at $\sim 0.80 \mathrm{~V}$ (named the butterfly) and the absence of contribution peaks in the featureless region below $\sim 0.4 \mathrm{~V}$ were already employed as diagnostic of solution cleanness and ordering of the crystal surface. For stepped electrodes, the coupled features at $\sim 0.126 \mathrm{~V}$ are attributed to hydrogen adsorption/desorption in step sites with (110) symmetry ${ }^{36}$ and, as can be seen in Fig. 1, its magnitude increases with the density of these sites. The nature of the reversible processes at the butterfly region is not yet completely elucidated. ${ }^{37}$ It should be stressed that the upper potential limits of the sweep were controlled for each case, to maintain a constant voltammetric profile in the low potential region.

\subsection{Influence of traces of dissolved CO on its pre-oxidation}

Fig. 2 compares the $\mathrm{CO}$ oxidation in presence and absence of small amounts of dissolved CO in the electrolyte for different Pt stepped surfaces. In these experiments, the dosing potential was fixed at $0.10 \mathrm{~V}$ and $\mathrm{CO}$ was bubbled into the solution for $5 \mathrm{~min}$ to fully cover the electrode surface. Afterwards, Ar was bubbled into the solution ( 150 $\mathrm{mL} / \mathrm{min}$ ) for 5, 10 and $60 \mathrm{~min}$ (indicated in Fig. 2). Data also include a control experiment in which the $\mathrm{CO}$ in solution was not purged at all (oxidation of bulk $\mathrm{CO}$, magenta line). For purposes of clarity, we show the beginning of the oxidation 
processes in major details in bottom panels for each electrode (Figs. 2D-2F). It is noteworthy to say that, after recording the CO stripping in the first cycle, the subsequent sweep evidenced the presence of small amounts of readsorbed CO (not shown). Moreover, after 5 min of purge, the voltammogram of the backward sweep already shown that the hydrogen adsorption on step sites (also not shown) was slightly suppressed, suggesting that there were also traces of CO in solution.

For $\operatorname{Pt}(13,13,12)$ the main CO oxidation peak is at $\sim 0.74 \mathrm{~V}$ (Fig. 2A). Moreover, when $\mathrm{Ar}$ is bubbled for $5 \mathrm{~min}$ (blue line) or $10 \mathrm{~min}$ (red line), a CO pre-oxidation signal is clearly discerned between $\sim 0.43 \mathrm{~V}$ and $\sim 0.66 \mathrm{~V}$, with a maximum at $\sim 0.55 \mathrm{~V}$ (Fig. 2D). On the other hand, when the solution is purged for 60 min (green line) the CO preoxidation signal becomes completely absent (Fig. 2D). The fact that the hydrogen region was fully blocked at the beginning of the stripping cycle (better visualized in bottom panels) evidences that both (111) terraces and (110) steps were fully covered by $\mathrm{CO}$ in all experiments. Results of Fig. 2 show that the CO pre-oxidation wave increases for shorter purging times, i.e., when the amount of dissolved CO is higher. For 5 min, 10 min and 60 min of purge, the CO coverage $\left(\theta_{\mathrm{CO} \text {,total }}\right.$ - corrected by the electric double layer) was estimated as $0.70,0.67$, and 0.63 . For the estimation of $\theta_{\mathrm{CO} \text {,total }}$ we calculate the potential of total zero charge ( $E_{\mathrm{ptzc}}$, in absence of $\mathrm{CO}$ in electrolyte) for each electrode by CO charge displacement. ${ }^{38}$ For Pt(13,13,12), $\operatorname{Pt}(776)$ and $\operatorname{Pt}(554)$, the $E_{\text {pztc }}$ values were of about $0.33,0.28$ and $0.24 \mathrm{~V}$ vs. RHE/V, respectively, in good agreement with previous results reported by Gómez et al. in $0.1 \mathrm{M} \mathrm{HClO}_{4}{ }^{39}$ For Pt(554) in $0.1 \mathrm{M}$ $\mathrm{HClO}_{4}$, Chen et al. ${ }^{10}$ found a $E_{\mathrm{ptzc}}$ of about 0.26 vs. RHE/V.

Now, comparing the onset potential for the CO oxidation in all cases, we can see that both CO pre-oxidation and oxidation of bulk CO start at very similar potentials for all purging conditions (c.f. blue and magenta lines in bottom panels). 
The observations for $\operatorname{Pt}(13,13,12)$ are representative of $\operatorname{Pt}(776)$ and $\operatorname{Pt}(554)$. For $\operatorname{Pt}(776)$, the CO coverage was $0.73,0.69$ and 0.64 for 5,10 and $60 \mathrm{~min}$ of purge, respectively. For $\mathrm{Pt}(554)$, the corresponding values of $\mathrm{CO}$ coverage were $0.71,0.70$ and 0.65. Our results are in perfect agreement with that of Chen et al., ${ }^{10}$ who found a $\theta_{\text {CO,total }}$ $\simeq 0.64$ on a $\operatorname{Pt}(554)$ electrode in acidic media. However, it is important to note that in this latter paper a slightly different approach ${ }^{10}$ was used, namely, CO gas was not bubbled through the solution, but introduced in the cell atmosphere over the meniscus formed between the solution and the electrode, and this CO-diluted solution was purged with Ar bubbling for at least 8 min. Under these conditions, the CO stripping does not exhibit a CO pre-oxidation wave in the experiments performed by Chen et al.. ${ }^{10}$ Finally, as expected, ${ }^{29}$ the potential of the main CO oxidation peak $\left(E_{\mathrm{p}}\right)$ decreases as the step density increases. We found $E_{\mathrm{p}}$ values of $\sim 0.74 \mathrm{~V}, \sim 0.72 \mathrm{~V}$ and $\sim 0.68 \mathrm{~V}$ for Pt(13,13,12), Pt(776) and Pt(554), respectively (see Fig. 2).

\subsection{Influence of the $\mathrm{CO}$ adsorption potential on its pre-oxidation}

Fig. 3 displays voltammograms of adsorbed CO oxidation after dosing at three different potentials. Aiming to allow a better visualization, the CO stripping curves were cut at $0.73 \mathrm{~V}$ in the figure. In these experiments, the $\mathrm{CO}$ adlayer was formed and the solution was purged with Ar for 10 min (this time was chosen since it provides a well-defined pre-oxidation wave, as seen in Fig. 2). As aforementioned, in these conditions a significant CO pre-oxidation wave appears between $\sim 0.45 \mathrm{~V}$ and $\sim 0.66 \mathrm{~V}$. However, the well-defined CO pre-oxidation signal only appears if the CO dosing potential is lower than $0.20 \mathrm{~V}$. This results is in line with data of Kita et al.. ${ }^{2}$ Although the onset potential of oxidation cannot be precisely determined by cyclic voltammetry, 
Fig. 3 shows that it begins earlier when $\mathrm{CO}$ is adsorbed at $0.06 \mathrm{~V}$ or $0.20 \mathrm{~V}$, in comparison to $\mathrm{CO}$ adsorbed at $0.35 \mathrm{~V}$. The adsorption of $\mathrm{CO}$ at $0.06,0.20$ and $0.35 \mathrm{~V}$ results in $\theta_{\mathrm{CO} \text {,total }}$ values of $0.70,0.66$ and 0.64 , respectively. These trends are similar to those reported by López-Cudero et al. ${ }^{9}$ for a defective Pt(111) electrode.

\subsection{CO oxidation on a Pt(111) surface rich of random defects}

Fig. 4 shows the CO stripping voltammograms (and the corresponding blank curves) for a well-ordered and a defective Pt(111) electrode. The defects were generated in a clean and initially well-ordered Pt(111) surface by cycling it at potentials up to 1.2 vs. RHE/V. Next, a CO adlayer was formed by dosing at $0.10 \mathrm{~V}$ on each electrode and the solution was purged with Ar for 30 min. Afterwards the CO adlayer was oxidized voltammetrically. Fig. 4B shows that the stripping of the CO adlayer on the defective electrode presents a negative shift $(\Delta E \simeq-40 \mathrm{mV})$ in the onset potential (red line) compared to the $\operatorname{Pt}(111)$ "defect-free” surface (black line). We can also see that, providing that the purging time is long enough to remove all non-adsorbed $\mathrm{CO}$, as in this case, there are no signals relative to the pre-oxidation wave. Here is noticeable that our results are different from those ${ }^{9}$ for a defect-rich $\mathrm{Pt}(111)$ electrode in $0.1 \mathrm{M} \mathrm{H}_{2} \mathrm{SO}_{4}$ in which a well-defined $\mathrm{CO}$ pre-oxidation signal between $\sim 0.5 \mathrm{~V}$ and $\sim 0.7 \mathrm{~V}$ was observed, even for "CO-free” experiments. However, it was specified that the solution was purged with $\mathrm{N}_{2}$ only for $20 \mathrm{~min}^{9}$ before the removal of non-adsorbed $\mathrm{CO}$, and we believe that such difference can explain the apparent discrepancies between both series of data.

\subsection{Identification of the catalytic active sites by partial oxidation of $\mathrm{CO}$ adlayer}


Fig. 5 shows cyclic voltammograms recorded during partial oxidation of a fully covered CO adlayer on $\mathrm{Pt}(776)$. After CO adsorption at $0.10 \mathrm{~V}$ and purging the solution with Ar for 18 min we used a lower potential limit to assure that CO was only partially oxidized, as described in a previous paper. ${ }^{30}$ Thus, the CO adlayer was gradually oxidized over several cycles. In Fig. 5A [Pt(776)], after the CO adlayer was formed, the potential was swept at $0.05 \mathrm{~V} \mathrm{~s}^{-1}$ from $0.10 \mathrm{~V}$ to $0.72 \mathrm{~V}$ (blue line, $1^{\text {st }}$ cycle), and then it was stepped back to $0.10 \mathrm{~V}$. (At the end of each partial CO stripping cycle, the electrode potential was always stepped back to $0.10 \mathrm{~V}$ ). During the $1^{\text {st }}$ scan the CO pre-oxidation wave appears in the range $0.41 \mathrm{~V}-0.64 \mathrm{~V}$ (peak potential at $\sim 0.56 \mathrm{~V}$ ). Before the $2^{\text {nd }}$ oxidation cycle (red line) no sites are recovered for hydrogen adsorption/desorption and the surface remain fully blocked. In the second cycle, a large current density is observed, implying that a large fraction of the adsorbed CO is stripped out in this cycle. Before the $3^{\text {rd }}$ cycle (green line) we can see that (111) terraces were completely available for hydrogen desorption, which means that CO was oxidized mainly from the terrace sites during the $2^{\text {nd }}$ cycle. Conversely, most (110) steps remain blocked, as can be inferred by the characteristic hydrogen desorption in $3^{\text {rd }}$ cycle. Further, we observe a distinct behavior for the oxidation of CO during this last cycle. Namely, CO is oxidized between $\sim 0.66 \mathrm{~V}$ and $\sim 0.76 \mathrm{~V}$ (maximum at $\sim 0.71 \mathrm{~V}$ ). According to these features we can assume that during the $3^{\text {rd }}$ cycle the oxidation of CO occurs exclusively on lines of (110) step sites separated by (111) terraces free of adsorbed CO. It is clear that this oxidation does not take place at low potential, despite that the terrace sites are free of adsorbed CO.

By comparing $1^{\text {st }}$ and $3^{\text {rd }}$ cycles in Fig. $5 \mathrm{~A}$ we can note that the CO pre-oxidation takes place at potentials $\sim 150 \mathrm{mV}$ lower than that for CO oxidation on (110) steps. After the $3^{\text {rd }}$ cycle, all remaining CO is oxidized. Afterwards, a new cycle was performed 
(black line) which served as a "blank" voltammetry, which is coincident to the obtained in absence of adsorbed CO.

Fig. 5B displays the cyclic voltammogram for the complete oxidation of a saturated CO adlayer on the same surface, followed by the final voltammetry which also superimposes that recorded before CO dosing. In this case, the main CO oxidation peak appears at $\sim 0.776 \mathrm{~V}$, i.e., about $60 \mathrm{mV}$ higher than the potential of $\mathrm{CO}$ oxidation on step sites alone. However, in this case the main peak $(\sim 0.776 \mathrm{~V})$ is due to $\mathrm{CO}$ oxidation on both (111) terraces and (110) steps. Qualitatively, all the observations for $\operatorname{Pt}(776)$ can be extrapolated for $\operatorname{Pt}(13,13,12)$ and $\mathrm{Pt}(554)$, as seen in Figs. 6A and 6C and their corresponding oxidations of a CO full adlayer (Figs. 6B and 6D).

The partial stripping of the initially fully covered CO adlayer was also performed on a “defect-free” Pt (111) surface (Fig. 7). This latter figure shows experiments for three CO coverages. Full coverage (magenta line) corresponds to the $\theta_{\mathrm{CO} \text {,total }} \simeq 0.70$ (estimated by the procedure described before and using a $E_{\mathrm{pztc}}=0.35 \mathrm{vs}$. RHE/V) and two intermediate $\mathrm{CO}$ coverage layers in which the hydrogen region was partially blocked ( 4 and $\sim 15 \%$, see figure). Fig. 7 shows that for CO coverage below saturation, the onset potential of oxidation is lower than that observed for a CO full coverage. This negative shift in the onset potential of CO oxidation at low CO coverage is in line with previous studies by Bilmes et al. ${ }^{40}$ (on polycrystalline Pt), Feliu et al. ${ }^{41}$ (on single crystals cooled in air and dosing at open circuit), and Lebedeva et al. ${ }^{29}$ [on $\mathrm{Pt}(111)]$ despite that in these works the low CO coverage was achieved by direct CO dosing, while we use partial CO stripping. The similarities of these results strongly suggest that the protocol for obtaining a partial CO coverage apparently does not affect its subsequent oxidation on a $\operatorname{Pt}(111)$ surface, providing that the adsorption potential is controlled. However, Feliu et al. $^{41}$ reported that if a low CO coverage is acquired at 
open circuit potential, its stripping voltammetry profile (in sulfuric acidic solution) differs from that obtained after partial stripping. This proves the influence of the adsorption step in the subsequent adlayer stripping. In order to get further insights into this subject we performed a chronoamperometric analysis.

\subsection{Partial removal of a CO adlayer by chronoamperometry}

For the analysis of the current vs. time curves, CO was admitted over the solution (in the atmosphere of the electrochemical cell) while the electrode potential was kept at $0.10 \mathrm{~V}$. Next, non-adsorbed CO was removed by purging the cell with Ar for $20 \mathrm{~min}$. Finally, two potential protocols were adopted. In the first one, the potential was stepped to a high value $\left(E_{\text {oxi }}\right)$ and maintained to remove the whole $\mathrm{CO}$ adlayer at once. Alternatively, the potential was alternated between $0.10 \mathrm{~V}$ and $E_{\text {oxi }}$ in several short sequences to allow removal of portions of adsorbed $\mathrm{CO}$ each time.

Fig. 8A shows the complete removal of the CO adlayer on a Pt(111) surface. The oxidation time of maximum current $\left(t_{\max }\right)$ is at $\sim 33 \mathrm{~s}$. A quick look at this transient reveals that it is very symmetrical around $t_{\max }$, as already reported by Lebedeva et $a l^{42}$. In Fig. 8B the CO adlayer was oxidized in two steps. The potential was first stepped to $0.69 \mathrm{~V}\left(E_{\text {oxi }}\right)$ and brought back to $0.10 \mathrm{~V}$ soon after $(\sim 6.5 \mathrm{~s}-$ at end of the exponential decay), where it remained for $30 \mathrm{~s}$; next, it was stepped again to $0.69 \mathrm{~V}$ until the complete oxidation of the remaining CO. In this case, if we disregard the time in which the system was kept at $0.10 \mathrm{~V}$ at the end of the first step the time of maximum current was $\sim 34.1 \mathrm{~s}$ (三63 s $-35.4 \mathrm{~s}+6.5 \mathrm{~s}$, see Fig. 8B), i.e., virtually the same time to that in the experiment displayed in Fig. 8A. It is noteworthy that even after potential steps the transient feature keeps the characteristic symmetry around $t_{\max }$. In experiments

(A) and (B), the oxidation charge densities were $\sim 409 \mu \mathrm{C} \mathrm{cm} \mathrm{cm}^{-2}$ and $\sim 402 \mu \mathrm{C} \mathrm{cm}{ }^{-2}$, 
respectively. The experimental conditions are extremely demanding: experiments were performed at several values of $E_{\text {oxi }}$, but we found situations in which the transients were either too fast (when $t_{\max }$ was very short) or too slow (in which $t_{\max }$ was poorly defined).

For Pt(554) a similar protocol was adopted with slight differences: $E_{\text {oxi }}$ was 0.67 $\mathrm{V}$ and several oxidation steps were applied instead of only two (Fig. 8D). Fig. 8C (single oxidation step; oxidation charge density $\sim 395 \mu \mathrm{C} \mathrm{cm}^{-2}$ ) shows that $t_{\max }$ is shorter than on $\operatorname{Pt}(111)$, but the oxidation persists until longer times than in the first case and the transient presents a tailing feature at the end of the oxidation. In Fig. 8C, we stepped the electrode potential back from $0.67 \mathrm{~V}$ to $0.10 \mathrm{~V}$ after $14 \mathrm{~s}$ (i.e., before the complete stripping of the $\mathrm{CO}$ adlayer). This procedure was adopted to register a cyclic voltammogram afterwards, as will be discussed later. Similar to Pt(111), Fig. 8D shows that when $\mathrm{CO}$ is oxidized in multiple stages on $\mathrm{Pt}(554)$ both the time required to reach the maximum current $\left(t_{\max }=22.7 \mathrm{~s}-20.9 \mathrm{~s}+7.8 \simeq 9.6 \mathrm{~s}\right)$ and the global oxidation profile were very similar to Fig. $8 \mathrm{C}\left(t_{\max } \simeq 9 \mathrm{~s}\right)$. As before, the potential was stepped back at $45 \mathrm{~s}$ before the CO adlayer was completely oxidized. In summary, the fact that the $\mathrm{CO}$ oxidation is performed in a single step or in multiple steps seems not to influence the kinetics of the overall process.

Figs. 9A and 9B show the corresponding cyclic voltammograms recorded after the last transients of Figs. 8C and 8D, respectively. The potential was cycled between 0.06 $\mathrm{V}$ and $0.80 \mathrm{~V}$, in order to observe the behavior of hydrogen sites blocked and the potential in which the stripping of the remaining $\mathrm{CO}$ adlayer occurs. Based on the discussion concerning Figs. 5 and 6 we infer that the CO remaining adlayer consists of CO adsorbed exclusively at (110) steps. This CO oxidizes only at high potentials, as already shown in Figs. 5 and 6. 


\subsection{In situ FTIR experiments}

Fig. 10 shows in situ FTIR spectra of CO adsorbed on a Pt(443) stepped electrode. As a member of the $\operatorname{Pt}(\mathrm{s})[(n-1)(111) \times(110)]$ family, it contains 7 atoms-wide terraces intercalated by monoatomic steps with (110) symmetry. The black line refers to the spectrum recorded at $0.15 \mathrm{~V}$ from the series registered when the surface is fully covered by CO. In this spectrum, ranging from low to high vibrational stretching frequencies, the broad band at $\sim 1600-1677 \mathrm{~cm}^{-1}$ is associated to the $\mathrm{O}-\mathrm{H}$ bending mode of water. ${ }^{33}$ The band at $\sim 1823 \mathrm{~cm}^{-1}$ is assigned to the stretching frequency of bridge bonded CO (hereafter denoted as $\left.\mathrm{CO}^{B}\right)^{7}$ at (111) terraces. Another band at $\sim 2060 \mathrm{~cm}^{-1}$ is assigned to the linearly bonded $\mathrm{CO}^{7}$ (hereafter denoted as $\mathrm{CO}^{L}$ ) at both (111) terraces and (110) step sites. In those experiments where CO selectively decorates (110) steps (blue line), a single band related to the CO stretching frequency appears at $\sim 2023 \mathrm{~cm}^{-1}$. This this band can be assigned to the $\mathrm{CO}^{L}$ in (110) step sites. No band due to $\mathrm{CO}^{B}$ in (110) sites is evident in this configuration (steps decorated by $\mathrm{CO}$ ).

For a complete CO coverage, from $0.05 \mathrm{~V}$ up to $0.40 \mathrm{~V}$, a Stark tuning plot gives a $\mathrm{d} v_{\mathrm{CO}}{ }^{i} / \mathrm{d} E \simeq 32 \mathrm{~cm}^{-1} \mathrm{~V}^{-1}$ for $\mathrm{CO}^{L}$ [in good agreement with $\sim 35 \mathrm{~cm}^{-1} \mathrm{~V}^{-1}$ previously reported for this electrode in $0.10 \mathrm{M} \mathrm{H}_{2} \mathrm{SO}_{4}{ }^{43}$ ] and $\mathrm{d} v_{\mathrm{CO}}{ }^{i} / \mathrm{d} E \simeq 114 \mathrm{~cm}^{-1} \mathrm{~V}^{-1}$ for $\mathrm{CO}^{B}$. In the experiment where CO is attached only on steps, $\mathrm{d} v_{\mathrm{CO}}{ }^{i} / \mathrm{d} E$ is $\sim 89 \mathrm{~cm}^{-1} \mathrm{~V}^{-1}$ (for $\mathrm{CO}^{L}$ ). A high $\mathrm{d} v_{\mathrm{CO}}{ }^{i} / \mathrm{d} E$ for $\mathrm{CO}$ on steps has also been observed by Kim et al. ${ }^{44-45}$ (low CO coverage obtained by CO dosing) for $\operatorname{Pt}(s)-[6(111) \times(100)] \equiv \operatorname{Pt}(755)$ and $\operatorname{Pt}(\mathrm{s})$ $[4(111) \times(100)] \equiv \operatorname{Pt}(533)$ but the origin for this high gradient at low coordinated sites is not yet understood. 
The comparison between the spectra in Fig. 10 shows that the band due to the $\mathrm{CO}^{L}$ on steps is clearly shifted $\left(\sim 37 \mathrm{~cm}^{-1}\right)$ to higher frequencies when the surface is fully covered by CO. As expected, for a full $\mathrm{CO}$ coverage $\mathrm{CO}^{L}$ on terrace sites strongly dominates the frequencies from $\mathrm{CO}^{L}$ on step sites due to dipole-dipole coupling. ${ }^{46-47}$ As a result, both $\mathrm{CO}^{L}$ bands merge into a single signal and becomes impossible to discriminate them, although a little tail is observed on the right side of $\mathrm{CO}^{L}$ band for the full coverage spectrum. ${ }^{46,48}$

\section{Discussion}

This work demonstrates that CO electro-oxidation reaction at stepped Pt surfaces occurs preferentially over (111) terrace domains at the expenses of step sites. Moreover, it shows that the CO pre-oxidation process only appears when four interconnected conditions are fulfilled: (1) adsorption of $\mathrm{CO}$ at potentials in $\mathrm{H}_{\text {upd }}$ region; (2) high CO coverage, which is secured by the (3) presence of traces of CO in solution; (4) steps on the surface. If these four requirements met, the occurrence of a reaction pathway at low potentials (denoted pre-oxidation) is reproducible.

Regarding the order in which sites are released, Figs. 5 and 6 clearly show that (111) terraces are the first sites to recovered for hydrogen adsorption/desorption in experiments of partial stripping of $\mathrm{CO}$ the adlayer. This observation means that on $\mathrm{Pt}$ stepped surfaces the over-potential required to oxidize CO on terrace domains is lower than that required for the reaction taking place on step sites. Therefore, the CO removal follows a strict hierarchy: CO is oxidized on (110) step sites only after it has been fully oxidized on (111) terraces. Similar trends can be inferred from data in Fig. 9 recorded after an incomplete $\mathrm{CO}$ adlayer oxidation. Namely, at the end of chronoamperometry CO remains only on the steps. This surprising result comes from the fact that such 
hierarchy is not accessible when a full CO adlayer is oxidized at once. Nevertheless, this apparent high catalytic activity of (111) terraces is not observed on a "defect-free" Pt(111) surface. This implies that the presence of steps influences the catalytic activity of terrace domains. One possible interpretation is that the steps change the energetic properties of terrace domains due to the Smoluchowski effect ${ }^{49}$ (total electron charge density). Hence, it is expected that a (111) terrace on a stepped surface have different chemical properties compared to an "infinite" (111) terrace on a perfect $\operatorname{Pt}(111)$ surface. $^{38,50}$ In this matter, long-range effect of steps in (111) terraces might be inferred from the examination of stretching frequencies of adsorbed $\mathrm{CO}$ on stepped surfaces containing terraces of different widths. ${ }^{51}$ In electrochemical environments, this topic has been addressed by Rodes et al. ${ }^{52}$ for adsorbed CO on a series of $\operatorname{Pt}(s)[(n-1)(111) \times(110)]$ and $\operatorname{Pt}(s)[n(111) \times(100)]$ stepped surfaces. It was pointed out that the density of steps markedly influences the stretching frequency of CO on (111) terraces as well as induces a potential dependence in $p(2 \times 2)-3 C O \rightarrow(\sqrt{ } 19 \times \sqrt{ } 19) R 23.4^{\circ}-13 C O$ phase transition. ${ }^{52}$ Such observations imply that stepped surfaces cannot be considered as a static combination of terraces and steps.

Turning back to our results, at $0.05 \mathrm{~V} \mathrm{~s}^{-1}$ the stripping of CO on $\operatorname{Pt}(111)$ shows an oxidation peak at $\sim 0.83 \mathrm{~V}$ (Fig. 4), whereas on stepped surfaces (Figs. 5 and 6 ) the potentials for the main CO oxidation peak are always lower, the shift being dependent on the terrace width. Thus, the combination between steps and terraces creates favorable conditions for CO oxidation on terraces at low potentials. Therefore, since the CO preoxidation process is not observed on "defect-free” Pt(111) (Fig. 4). At a first glance, one could think that it is a feature coming from the presence of steps on the surface. The mere presence of small amounts of $\mathrm{CO}$ in solution is a condition that ensures a high CO coverage, but we think that the pre-oxidation is not due to the electro-oxidation 
of CO from the solution, because even when small amounts of CO in solution was present (Fig. 3), the pre-oxidation process does not appear if the low adsorption potential condition (which ensures higher CO coverage degree) is not satisfied. It seems more likely that the pre-oxidation is a consequence of some CO that is weakly bonded to the surface, but it is worthy to emphasize that CO cannot be bonded anywhere on surface. However, as evidenced in Fig. 2, the pre-oxidation of CO does not take place on (110) step sites, i.e., there is no oxidation of CO on the top side of (110) steps, since in that experiment the (110) step sites were fully covered by CO, and the pre-oxidation peak was completely absent. Indeed, our results show that, regardless the surface, the pre-oxidation peaks appear around $0.58 \mathrm{~V}$ whereas the oxidation of $\mathrm{CO}$ on step sites only takes place at $\sim 0.72 \mathrm{~V}$. These findings convincingly demonstrate that the steps are not the most surface active sites, at least for $\mathrm{CO}$ electro-oxidation. This implies that equation (2) is not valid for the CO oxidation at low potentials, where CO preferentially oxidizes over (111) terrace sites. For $\operatorname{Pt}(\mathrm{s})[(n-1)(111) \times(110)]$ vicinal surfaces, recently imaged by using in situ STM (Scanning Tunneling Microscopy), Inukai et al. ${ }^{53}$ suggested that the "most active sites" to the oxidation of bulk CO are disordered (111) step sites, e.g. kink sites. They observed that such sites are stable only at potentials lower than $\sim 0.6 \mathrm{~V}$ vs. RHE in presence of $\mathrm{CO}$ in the electrolyte. The authors also showed that when the electrode potential was cycled beyond that value, the observed loss of activity was directly linked to the morphological changes of the step sites. For experiments of CO oxidation performed on defect-rich Pt(100) surfaces cooled in air or Ar, Rudnev et al. also had identified kink and step sites as the highly active ones. ${ }^{54}$ However, by using a kinked $\operatorname{Pt}(875)(\equiv \operatorname{Pt}(\mathrm{s})-[5(111) \times 3(110) \times(100)])$ surface, we have found that kink sites are not the most active ones in CO electro-oxidation. ${ }^{30}$ Interestingly, all these works and others evidence that low coordinated sites 
(step/kinks, ${ }^{9,}{ }^{15,53-54}$ Pt ad-islands ${ }^{55}$ ) should be present on surface in order to ensure that the reaction pathway arises at low potentials. The exact nature of these sites is still an unsolved question, but it is likely that active sites responsible for the CO pre-oxidation might be the low part of the steps, which are terrace sites.

By using a class of Pt stepped surfaces containing (111) terraces periodically broken by (100) monoatomic steps, Yates et al. ${ }^{56-57}$ showed that in solid/gas interfaces CO oxidizes preferentially on (111) terraces neighbor to steps with $\mathrm{O}_{\mathrm{ads}}$. In this model, step sites act as centers for nucleation of $\mathrm{O}_{\text {ads }}$ (from $\mathrm{O}_{2}$ dissociation). Considering that $\mathrm{OH}_{\text {ads }}$ (from water dissociation) is the preferential species on the steps, Lebedeva et $a l{ }^{58}$ extrapolated the model of Yates et al. to the electrochemical stripping of CO. Based on this finding, the authors concluded that the role of steps is to promote the formation of $\mathrm{OH}_{\text {ads. }}$ Additionally, it was proposed ${ }^{58}$ that during the oxidation $\mathrm{CO}$ adlayer $\mathrm{CO}_{\mathrm{ads}}$ could diffuses along (111) terraces to the most active sites, near $\mathrm{OH}_{\mathrm{ads}}$ on steps. However, as stressed above, the role of steps seems not to be simply related as centers of nucleation of oxygen-containing species for $\mathrm{CO}$ oxidation on terraces. Moreover, data in Fig. 5 and 6 allow us to infer about the dynamics/mobility of adsorbed CO during its oxidation. These figures show that once the pre-oxidation adlayer was removed, in subsequent cycles all remaining $\mathrm{CO}_{\mathrm{ads}}$ molecules were oxidized at higher potentials than those of the CO pre-oxidation range. Assuming that the sites responsible for the pre-oxidation of $\mathrm{CO}$ are the most active ones, this observation means that even when these sites are available, there is no significant displacement of $\mathrm{CO}_{\mathrm{ads}}$ toward these "preferred" places, likely because this process is apparently not favorable, once there is a hierarchy for the occupancy of sites by CO, which is opposed to the CO site release. ${ }^{30}$ This evidence suggests that at least to some extent, the activation of particular surface sites controls the order in which sites are 
released during $\mathrm{CO}$ oxidation on Pt stepped surfaces. A similar behavior has been pointed out for CO oxidation on Pt stepped electrodes in alkaline medium. ${ }^{30}$ Such observation brings important implications on the $\mathrm{CO}_{\text {ads }}$ mobility during its oxidation in acidic media too. Namely, although the replacement of a previously adsorbed CO molecule by another CO (coming from the solution) is possible, once adsorbed, during its oxidation process, CO molecules apparently do not diffuse over the surface toward most active sites, even when a more active site is available.

The observations about the apparent immobility of $\mathrm{CO}_{\text {ads }}$ during adlayer stripping are reinforced by the analysis of Fig. 8 (current vs. time curves). The chronoamperometric profiles in Figs. 8B and 8D may be related to the distribution of reactant partners on the surface during successive potential steps. Thus, is likely that activated water ${ }^{7}$ (or $\mathrm{OH}_{\mathrm{ads}}$ ) is formed at a potential in which $\mathrm{CO}$ oxidizes (in the present case at $0.67 \mathrm{~V}$ or $0.69 \mathrm{~V}$ ). Afterwards, when the potential is stepped back to $0.10 \mathrm{~V}$, the oxygen-containing species population formed at $E_{\mathrm{CO}}$ oxidation is completely stripped out. In this process, sites are released, which come to be occupied by adsorbed hydrogen at $0.10 \mathrm{~V}$. Thus, if $\mathrm{CO}_{\mathrm{ads}}$ had high mobility over the surface during its oxidation, or if there was redistribution of reactants on electrode surface at $0.10 \mathrm{~V}$, the release of sites previously occupied with oxygen-containing species could imply changes in patterns of surface distribution of the remaining $\mathrm{CO}_{\text {ads }}$. Surprisingly, we observed that after the $\mathrm{CO}$ oxidation is resumed, it continues exactly where it was stopped before, regardless the sequence of potential steps applied (compare Figs. 8C and 8D). This interpretation agrees with results showed in Figs. 5 and 6, in which after active sites becomes free, they are not occupied by the remaining $\mathrm{CO}_{\text {ads }}$ molecules, likely because this process might be too slow to take place in the time scale of our experiments. Therefore, since the reaction kinetics depends on the spatial distribution of the reacting partners on the 
surface, $^{59-61}$ our results allow us to consider that the organization (distribution) of remaining adsorbed CO layer is not significantly affected either after potential steps or partial stripping voltammetry.

One possibility to explain the current vs. time behavior observed in Fig. 8 is that $\mathrm{CO}_{\mathrm{ads}}$ forms large domains (islands) in which the $\mathrm{CO}$ oxidation starts by the edges of these islands, as previously discussed in ${ }^{62-64}$. Thus, after each potential step the reaction is resumed when oxygen-containing species combines with $\mathrm{CO}_{\mathrm{ads}}$ from the edge of the islands, which were left unchanged since the last oxidation step. Lastly, ours results can be satisfactorily explained by considering the absence of $\mathrm{CO}_{\text {ads }}$ mobility during its oxidation.

\section{Conclusions}

In this work, some important findings are presented and discussed for the first time regarding the oxidation of $\mathrm{CO}$ on $\mathrm{Pt}(\mathrm{s})[(n-1)(111) \times(110)]$ in acidic medium:

(1) Providing that experimental conditions are controlled (i.e. CO adsorption in the $\mathrm{H}_{\text {upd }}$ region, high CO coverage, presence of steps on the surface), the CO pre-oxidation is related to the presence (trace amount) of dissolved CO in solution. The pre-oxidation of CO seems to be not due to its oxidation in step sites, although the presence of steps is a sine qua non condition for the occurrence of pre-oxidation. We assume that the sites responsible for the $\mathrm{CO}$ pre-oxidation are likely those atoms at the bottom of the steps with weakly bonded CO. Also, it seems that the initial activation of some particular sites controls the whole process.

(2) The intrinsic catalytic activity on step sites is lower than that of the sites responsible for the CO pre-oxidation. Indeed, (111) terraces seem to constitute the most 
active domains for the electro-oxidation of $\mathrm{CO}$, since $\mathrm{CO}$ on step sites is oxidized only after all CO molecules have been oxidized over (111) terraces.

(3) The present analysis implies that CO behaves almost as a motionless species during its oxidation, likely because its surface diffusion coefficient make the diffusion process too slow to take place in the time scale of our experiments. Therefore, after the "most active sites" to be free due to the partial CO adlayer oxidation, such "most active sites” apparently does not become reoccupied by new CO molecules from the remaining CO layer. It seems more likely that domains of oxygen-containing species $\left[\left(\mathrm{H}_{2} \mathrm{O}\right)_{\text {activated }}\right]$ growing around $\mathrm{CO}$ islands rather than $\mathrm{CO}$ shifts to the most active sites.

Acknowledgements: M.J.S. Farias is grateful to the CNPq (Brazil) (grants: 200390/2011-2 and 313402/2013-2) for the financial support. G.A. Camara acknowledges financial assistance from CNPq (grants: 305494/2012-0 and 405695/2013-6) and FUNDECT (grant: 23/200.583/2012). J.M. Feliu thanks to the MINECO (Spain) project CTQ2013-44083-P. 


\section{References}

1. Grambow, L.; Bruckenstein, S., Mass Spectrometric Investigation of the Electrochemical Behavior of Adsorbed Carbon Monoxide at Platinum in $0.2 \mathrm{M}$ Sulphuric Acid. Electrochim. Acta 1977, 22, 377-383.

2. $\quad$ Kita, H.; Shimazu, K.; Kunimatsu, K., Electrochemical Oxidation of CO on Pt in Acidic and Alkaline Solutions: Part I. Voltammetric Study on the Adsorbed Species and Effects of Aging and Sn(IV) Pretreatment. J. Electroanal. Chem. 1988, 241, 163-179.

3. Iwasita, T., Methanol and CO Electrooxidation. In Handbook of Fuel Cells, John Wiley \& Sons, Ltd: 2010.

4. Iwasita, T.; Camara, G. A., Chapter 2 - Contributions of External Reflection Infrared Spectroscopy to Study the Oxidation of Small Organic Molecules. In In-Situ Spectroscopic Studies of Adsorption at the Electrode and Electrocatalysis, Sun, S.-G.; Christensen, P. A.; Wieckowski, A., Eds. Elsevier Science B.V.: Amsterdam, 2007; pp 33-61.

5. Marković, N. M.; Grgur, B. N.; Lucas, C. A.; Ross, P. N., Electrooxidation of $\mathrm{CO}$ and $\mathrm{H}_{2} / \mathrm{CO}$ Mixtures on Pt(111) in Acid Solutions. J. Phys. Chem. B 1999, 103, 487-495.

6. Santos, E.; Leiva, E. P. M.; Vielstich, W., CO Adsorbate on Pt(111) Single Crystal Surfaces. Electrochim. Acta 1991, 36, 555-561.

7. Batista, E. A.; Iwasita, T.; Vielstich, W., Mechanism of Stationary Bulk CO Oxidation on Pt(111) Electrodes. J. Phys. Chem. B 2004, 108, 14216-14222.

8. Akemann, W.; Friedrich, K. A.; Stimming, U., Potential-Dependence of CO Adlayer Structures on $\mathrm{Pt}(111)$ Electrodes in Acid Solution: Evidence for a Site Selective Charge Transfer. J. Chem. Phys. 2000, 113, 6864-6874.

9. López-Cudero, A.; Cuesta, A.; Gutiérrez, C., Potential Dependence of the Saturation CO Coverage of Pt Electrodes: The Origin of the Pre-Peak in CO-Stripping Voltammograms. Part 1: Pt(111). J. Electroanal. Chem. 2005, 579, 1-12.

10. Chen, Q. S.; Berna, A.; Climent, V.; Sun, S. G.; Feliu, J. M., Specific Reactivity of Step Sites Towards CO Adsorption and Oxidation on Platinum Single Crystals Vicinal to Pt(111). Phys. Chem. Chem. Phys. 2010, 12, 11407-11416.

11. Chen, Q. S.; Vidal-Iglesias, F. J.; Solla-Gullón, J.; Sun, S. G.; Feliu, J. M., Role of Surface Defect Sites: From Pt Model Surfaces to Shape-Controlled Nanoparticles. Chem. Sci. 2012, 3, 136-147.

12. Cuesta, A.; Couto, A.; Rincón, A.; Perez, M.; Lopez-Cudero, A.; Gutierrez, C., Potential Dependence of the Saturation CO Coverage of Pt Electrodes: The Origin of the Pre-Peak in CO-Stripping Voltammograms. Part 3: Pt (Poly). J. Electroanal. Chem. 2006, 586, 184-195.

13. Samjeské, G.; Komatsu, K.-i.; Osawa, M., Dynamics of CO Oxidation on a Polycrystalline Platinum Electrode: A Time-Resolved Infrared Study. J. Phys. Chem. C 2009, 113, 10222-10228.

14. Yan, Y.-G.; Yang, Y.-Y.; Peng, B.; Malkhandi, S.; Bund, A.; Stimming, U.; Cai, W.-B., Study of CO Oxidation on Polycrystalline Pt Electrodes in Acidic Solution by ATR-SEIRAS. J. Phys. Chem. C 2011, 115, 16378-16388.

15. López-Cudero, A.; Cuesta, Á.; Gutiérrez, C., Potential Dependence of the Saturation CO Coverage of Pt Electrodes: The Origin of the Pre-Peak in CO-Stripping Voltammograms. Part 2: Pt (100). J. Electroanal. Chem. 2006, 586, 204-216.

16. Marković, N. M.; Schmidt, T. J.; Grgur, B. N.; Gasteiger, H. A.; Behm, R. J.; Ross, P. N., Effect of Temperature on Surface Processes at the Pt(111) - Liquid 
Interface: Hydrogen Adsorption, Oxide Formation, and CO Oxidation. J. Phys. Chem. B 1999, 103, 8568-8577.

17. Jung, C.; Kim, J.; Rhee, C. K., Electrochemical Scanning Tunneling Microscopic Observation of the Preoxidation Process of CO on Pt(111) Electrode Surface. Langmuir 2007, 23, 9495-9500.

18. Cuesta, A., The Oxidation of Adsorbed CO on Pt(100) Electrodes in the PrePeak Region. Electrocatalysis 2010, 1, 7-18.

19. Wieckowski, A.; Rubel, M.; Gutiérrez, C., Reactive Sites in Bulk Carbon Monoxide Electro-Oxidation on Oxide-Free Platinum(111). J. Electroanal. Chem. 1995, 382, 97-101.

20. Ertl, G.; Neumann, M.; Streit, K., Chemisorption of CO on the Pt (111) Surface. Surf. Sci. 1977, 64, 393-410.

21. Leiva, E. P. M.; Santos, E.; Iwasita, T., The Effect of Adsorbed Carbon Monoxide on Hydrogen Adsorption and Hydrogen Evolution on Platinum. $J$. Electroanal. Chem. 1986, 215, 357-367.

22. Lebedeva, N. P.; Koper, M. T. M.; Feliu, J. M.; Van Santen, R. A., Role of Crystalline Defects in Electrocatalysis: Mechanism and Kinetics of CO Adlayer Oxidation on Stepped Platinum Electrodes. J. Phys. Chem. B 2002, 106, 12938-12947.

23. Koper, M. T. M., Structure Sensitivity and Nanoscale Effects in Electrocatalysis. Nanoscale 2011, 3, 2054-2073.

24. Bandarenka, A. S.; Koper, M. T. M., Structural and Electronic Effects in Heterogeneous Electrocatalysis: Toward a Rational Design of Electrocatalysts. J. Catal. 2013, 308, 11-24.

25. Gilman, S., The Mechanism of Electrochemical Oxidation of Carbon Monoxide and Methanol on Platinum. II. The "Reactant-Pair" Mechanism for Electrochemical Oxidation of Carbon Monoxide and Methanol. J. Phys. Chem. 1964, 68, 70-80.

26. Wang, H.; Jusys, Z.; Behm, R. J.; Abruña, H. D., New Insights into the Mechanism and Kinetics of Adsorbed CO Electrooxidation on Platinum: Online Mass Spectrometry and Kinetic Monte Carlo Simulation Studies. J. Phys. Chem. C 2012, 116, 11040-11053.

27. Inkaew, P.; Zhou, W.; Korzeniewski, C., CO Monolayer Oxidation at Pt(100) Probed by Potential Step Measurements in Comparison to Pt(111) and Pt Nanoparticle Catalyst. J. Electroanal. Chem. 2008, 614, 93-100.

28. Rudnev, A. V.; Kuzume, A.; Fu, Y.; Wandlowski, T., CO Oxidation on Pt(100): New Insights Based on Combined Voltammetric, Microscopic and Spectroscopic Experiments. Electrochim. Acta 2014, 133, 132-145.

29. Lebedeva, N. P.; Koper, M. T. M.; Herrero, E.; Feliu, J. M.; Van Santen, R. A., Cooxidation on Stepped Pt[n(111) $\times(111)]$ Electrodes. J. Electroanal. Chem. 2000, 487, 37-44.

30. Farias, M. J. S.; Herrero, E.; Feliu, J. M., Site Selectivity for CO Adsorption and Stripping on Stepped and Kinked Platinum Surfaces in Alkaline Medium. J. Phys. Chem. C 2013, 117, 2903-2913.

31. Clavilier, J.; Armand, D.; Sun, S. G.; Petit, M., Electrochemical Adsorption Behaviour of Platinum Stepped Surfaces in Sulphuric Acid Solutions. J. Electroanal. Chem. 1986, 205, 267-277.

32. Lang, B.; Joyner, R. W.; Somorjai, G. A., Low Energy Electron Diffraction Studies of High Index Crystal Surfaces of Platinum. Surf. Sci. 1972, 30, 440-453.

33. Iwasita, T.; Nart, F. C., In Situ Infrared Spectroscopy at Electrochemical Interfaces. Prog. Surf. Sci. 1997, 55, 271-340. 
34. Greenler, R. G., Infrared Study of Adsorbed Molecules on Metal Surfaces by Reflection Techniques. J. Chem. Phys. 1966, 44, 310-315.

35. Hansen, W. N., Electric Fields Produced by the Propagation of Plane Coherent Electromagnetic Radiation in a Stratified Medium. J. Opt. Soc. Am. 1968, 58, 380-388.

36. Clavilier, J.; El Achi, K.; Rodes, A., In Situ Probing of Step and Terrace Sites on Pt(s)-[n(111)×(111)] Electrodes. Chem. Phys. 1990, 141, 1-14.

37. Berná, A.; Climent, V.; Feliu, J. M., New Understanding of the Nature of OH Adsorption on Pt(111) Electrodes. Electrochem. Comm. 2007, 9, 2789-2794.

38. Climent, V.; Gómez, R.; Feliu, J. M., Effect of Increasing Amount of Steps on the Potential of Zero Total Charge of Pt(111) Electrodes. Electrochim. Acta 1999, 45, 629-637.

39. Gómez, R.; Climent, V.; Feliu, J. M.; Weaver, M. J., Dependence of the Potential of Zero Charge of Stepped Platinum (111) Electrodes on the Oriented StepEdge Density: Electrochemical Implications and Comparison with Work Function Behavior. J. Phys. Chem. B 2000, 104, 597-605.

40. Bilmes, S.; De Tacconi, N.; Arvia, A., The Electrooxidation of Chemisorbed CO on Polycrystalline Platinum: A Mechanistic Interpretation of the Anodic Current Peak Multiplicity. J. Electroanal. Chem. 1984, 164, 129-143.

41. Feliu, J. M.; Orts, J. M.; Femandez-Vega, A.; Aldaz, A.; Clavilier, J., Electrochemical Studies in Sulphuric Acid Solutions of Adsorbed CO on Pt (111) Electrodes. J. Electroanal. Chem. 1990, 296, 191-201.

42. Lebedeva, N. P.; Koper, M. T. M.; Feliu, J. M.; Van Santen, R. A., Mechanism and Kinetics of the Electrochemical CO Adlayer Oxidation on Pt(111). J. Electroanal. Chem. 2002, 524-525, 242-251.

43. Farias, M. J. S.; Busó-Rogero, C.; Gisbert, R.; Herrero, E.; Feliu, J. M., Influence of the CO Adsorption Environment on Its Reactivity with (111) Terrace Sites in Stepped Pt Electrodes under Alkaline Media. J. Phys. Chem. C 2014, 118, 19251934.

44. Kim, C. S.; Korzeniewski, C.; Tornquist, W. J., Site Specific co-Adsorption at $\mathrm{Pt}(335)$ as Probed by Infrared Spectroscopy: Structural Alterations in the CO Adlayer under Aqueous Electrochemical Conditions. J. Chem. Phys. 1994, 100, 628-630.

45. Kim, C. S.; Korzeniewski, C., Vibrational Coupling as a Probe of Adsorption at Different Structural Sites on a Stepped Single-Crystal Electrode. Anal. Chem. 1997, 69, 2349-2353.

46. Persson, B. N. J.; Ryberg, R., Vibrational Interaction between Molecules Adsorbed on a Metal Surface: The Dipole-Dipole Interaction. Phys. Rev. B 1981, 24, 6954-6970.

47. Severson, M. W.; Stuhlmann, C.; Villegas, I.; Weaver, M. J., Dipole-Dipole Coupling Effects Upon Infrared Spectroscopy of Compressed Electrochemical Adlayers: Application to the Pt(111)/CO System. J. Chem. Phys. 1995, 103, 9832-9843.

48. Persson, B. N. J.; Hoffmann, P. M., Vibrational Phase Relaxation at Surfaces: The Role of Lateral Interaction. J. Electron Spectrosc. Relat. Phenom. 1987, 45, 215225.

49. Smoluchowski, R., Anisotropy of the Electronic Work Function of Metals. Phys. Rev. 1941, 60, 661-674.

50. Climent, V.; Attard, G. A.; Feliu, J. M., Potential of Zero Charge of Platinum Stepped Surfaces: A Combined Approach of CO Charge Displacement and $\mathrm{N}_{2} \mathrm{O}$ Reduction. J. Electroanal. Chem. 2002, 532, 67-74. 
51. Xu, J.; Yates Jr, J. T., Terrace Width Effect on Adsorbate Vibrations: A Comparison of Pt(335) and Pt(112) for Chemisorption of CO. Surf. Sci. 1995, 327, 193201.

52. Rodes, A.; Gómez, R.; Feliu, J. M.; Weaver, M. J., Sensitivity of Compressed Carbon Monoxide Adlayers on Platinum(111) Electrodes to Long-Range Substrate Structure: Influence of Monoatomic Steps. Langmuir 2000, 16, 811-816.

53. Inukai, J.; Tryk, D. A.; Abe, T.; Wakisaka, M.; Uchida, H.; Watanabe, M., Direct STM Elucidation of the Effects of Atomic-Level Structure on Pt(111) Electrodes for Dissolved CO Oxidation. J. Am. Chem. Soc. 2013, 135, 1476-1490.

54. Rudnev, A. V.; Wandlowski, T., An Influence of Pretreatment Conditions on Surface Structure and Reactivity of Pt(100) Towards CO Oxidation Reaction. Russ J Electrochem 2012, 48, 259-270.

55. Strmcnik, D. S.; Tripkovic, D. V.; Van Der Vliet, D.; Chang, K. C.; Komanicky, V.; You, H.; Karapetrov, G.; Greeley, J. P.; Stamenkovic, V. R.; Marković, N. M., Unique Activity of Platinum Adislands in the CO Electrooxidation Reaction. J. Am. Chem. Soc. 2008, 130, 15332-15339.

56. Yates, J. T., Surface Chemistry at Metallic Step Defect Sites. J. Vac. Sci. Technol. A 1995, 13, 1359-1367.

57. Xu, J.; Yates, J. T., Catalytic Oxidation of Co on Pt(335): A Study of the Active Site. J. Chem. Phys. 1993, 99, 725-732.

58. Lebedeva, N. P.; Rodes, A.; Feliu, J. M.; Koper, M. T. M.; Van Santen, R. A., Role of Crystalline Defects in Electrocatalysis: CO Adsorption and Oxidation on Stepped Platinum Electrodes as Studied by in situ Infrared Spectroscopy. J. Phys. Chem. B 2002, 106, 9863-9872.

59. Ertl, G., Heterogeneous Catalysis on the Atomic Scale. Chem. Rec. 2001, 1, 3345.

60. Zaera, F., Kinetics of Chemical Reactions on Solid Surfaces: Deviations from Conventional Theory. Acc. Chem. Res. 2002, 35, 129-136.

61. Zhdanov, V. P., Impact of Surface Science on the Understanding of Kinetics of Heterogeneous Catalytic Reactions. Surf. Sci. 2002, 500, 966-985.

62. Furuya, N.; Motto, S.; Kunimatsu, K., Vibrational Spectroscopy on Platinum Single-Crystal Electrodes: Part I. In-Situ Infrared Spectroscopic Studies of the Adsorption and Oxidation of CO on Pt (111) in Sulphuric Acid. J. Electroanal. Chem. 1988, 239, 347-360.

63. Chang, S. C.; Weaver, M. J., Coverage- Dependent Dipole Coupling for Carbon Monoxide Adsorbed at Ordered Platinum (111)-Aqueous Interfaces: Structural and Electrochemical Implications. J. Chem. Phys. 1990, 92, 4582.

64. Chen, Y.; Heinen, M.; Jusys, Z.; Behm, R., Potential-Induced $\mathrm{CO}_{\mathrm{ad}}$ Island Formation on a Platinum Thin-Film Electrode in the $\mathrm{H}_{\text {-UPD }}$ Potential Region. J. Phys. Chem. C 2007, 111, 435-438. 


\section{Figure Captions}

Figure 1. Cyclic voltammograms of platinum single crystals (indicated) in $0.1 \mathrm{M}$ $\mathrm{HClO}_{4}$ and hard sphere model of corresponding surfaces. Data recorded at $0.05 \mathrm{~V} \mathrm{~s}^{-1}$.

Figure 2. Effect of dissolved CO on its pre-oxidation on Pt stepped surfaces in $0.1 \mathrm{M}$ $\mathrm{HClO}_{4}$. A. Pt $(13,13,12)$; B. Pt (776); C. (Pt (554). The panels D, E and F correspond to the panels A, B and C, respectively, presented on an extended scale. After adsorption at $0.10 \mathrm{~V}, \mathrm{CO}$ was removed from the solution for different times (indicated). The oxidation of bulk CO and a blank voltammetry were showed for comparison. Data recorded at $0.02 \mathrm{~V} \mathrm{~s}^{-1}$.

Figure 3. Influence of $\mathrm{CO}$ adsorption potential on its pre-oxidation on Pt stepped surfaces in $0.1 \mathrm{M} \mathrm{HClO}_{4}$. Sweep rate $0.05 \mathrm{~V} \mathrm{~s}^{-1}$.

Figure 4. (A) Cyclic voltammograms for a well-ordered Pt (111) (black line) and an intentionally defective Pt (111) (red line) electrode in $0.1 \mathrm{M} \mathrm{HClO}_{4}$; (B) Stripping of CO performed on the same surfaces. Sweep rate $0.05 \mathrm{~V} \mathrm{~s}^{-1}$.

Figure 5. A. Cyclic voltammetry in presence of a $\mathrm{CO}$ adlayer which is oxidized in successive cycles (indicated) by selecting a low upper potential limit on a $\mathrm{Pt}$ (776) surface in presence of $0.1 \mathrm{M} \mathrm{HClO}_{4}$. B. Oxidation of a full $\mathrm{CO}$ adlayer in a single sweep. In A and B a blank voltammetry is showed for comparison. Data recorded at $0.05 \mathrm{~V} \mathrm{~s}^{-1}$.

Figure 6. A. Cyclic voltammetry in presence of a $\mathrm{CO}$ adlayer obtained as in Fig. 5 for Pt $(13,13,12)$ and $(554)$. Bottom panels (C and D) show the oxidation of a full CO adlayer in a single sweep. In all cases a blank voltammetry was included for comparison. Data recorded at $0.05 \mathrm{~V} \mathrm{~s}^{-1}$. 
Figure 7. Oxidation of $\mathrm{CO}$ at different CO coverage (indicated) on a "defect-free" $\mathrm{Pt}(111)$ electrode in $0.1 \mathrm{M} \mathrm{HClO}_{4}$. The partial coverages were acquired by partial stripping of a full CO adlayer. Sweep rate $0.05 \mathrm{Vs}^{-1}$.

Figure 8. Potential step experiments of CO oxidation on: A and B. Pt (111); C and D. Pt (554). CO was adsorbed at $0.10 \mathrm{~V}$, and non-adsorbed CO was removed for $20 \mathrm{~min}$. The left panels show the oxidation of CO performed in a single step for Pt (111) and Pt (554), respectively. The right panels show successive potential steps. In B a partial CO oxidation was achieved by applying the sequence $0.10 \mathrm{~V} \rightarrow 0.69 \mathrm{~V} \rightarrow 0.10 \mathrm{~V}$ on Pt (111). In D the sequence of step potentials $(0.10 \mathrm{~V} \rightarrow 0.67 \mathrm{~V} \rightarrow 0.10 \mathrm{~V} \rightarrow 0.67 \mathrm{~V} \ldots)$ was performed on $\mathrm{Pt}$ (554) All the experiments were made in $0.1 \mathrm{M} \mathrm{HClO}_{4}$.

Figure 9. Cyclic voltammetries in presence of residual CO that has persisted on the surface after the experiments of Figs. 8C and 8D. Sweep rate $0.05 \mathrm{Vs}^{-1}$.

Figure 10. In situ FTIR spectra recorded at $0.15 \mathrm{~V}$ with different CO coverage on a $\mathrm{Pt}(443)$ stepped crystal in $0.1 \mathrm{M} \mathrm{HClO}_{4}$. The reference potential was collected at $0.90 \mathrm{~V}$. 


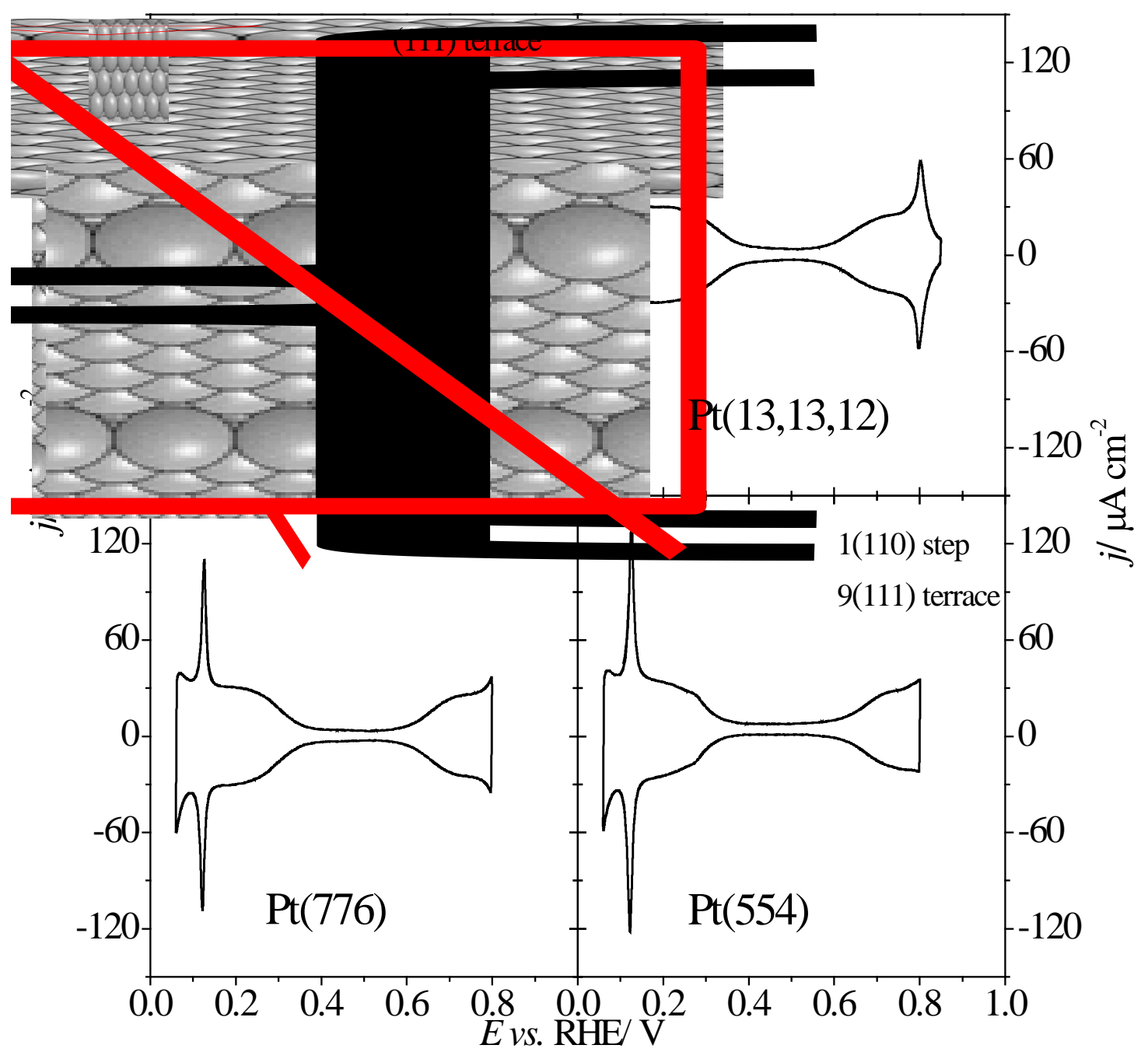

Figure 1, Farias et al. 


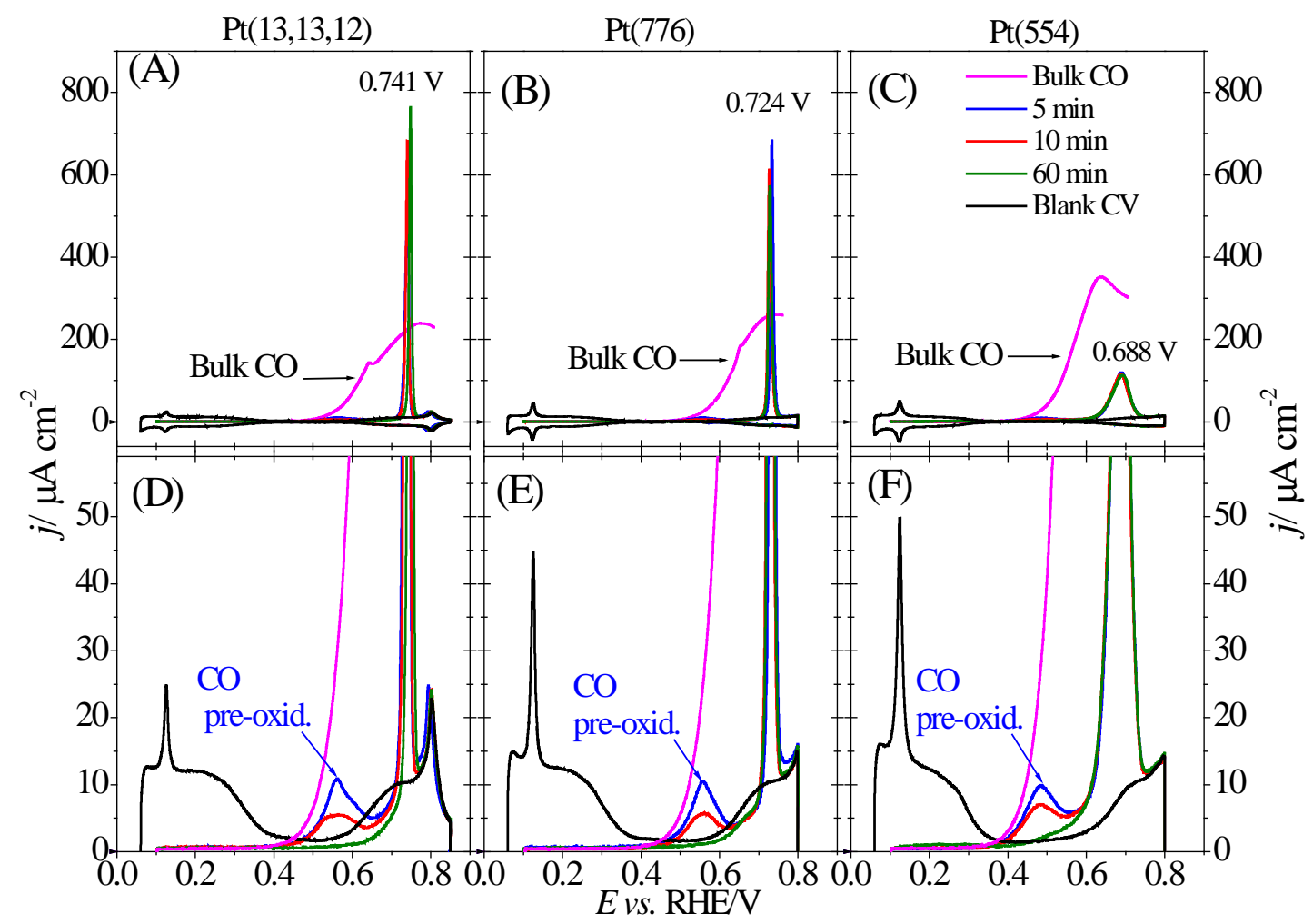

Figure 2, Farias et al. 


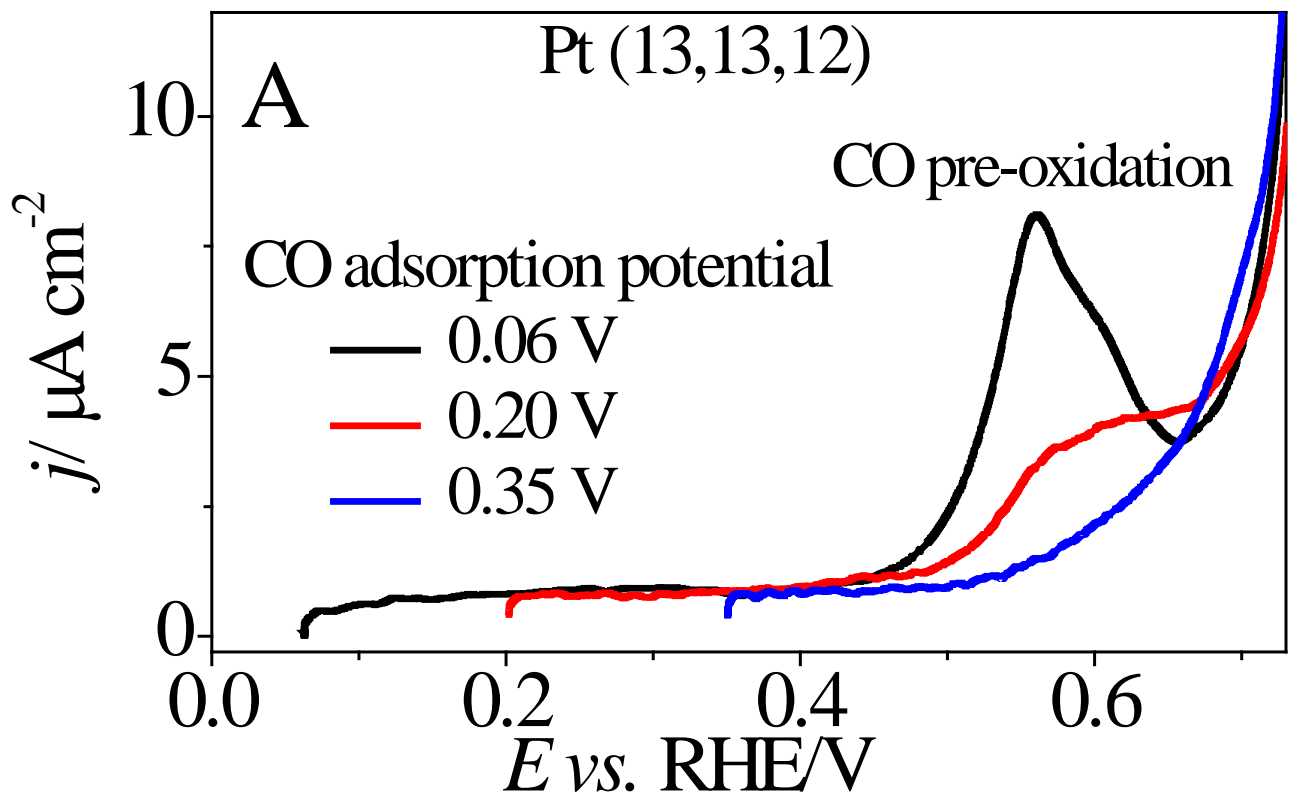

Figure 3, Farias et al. 


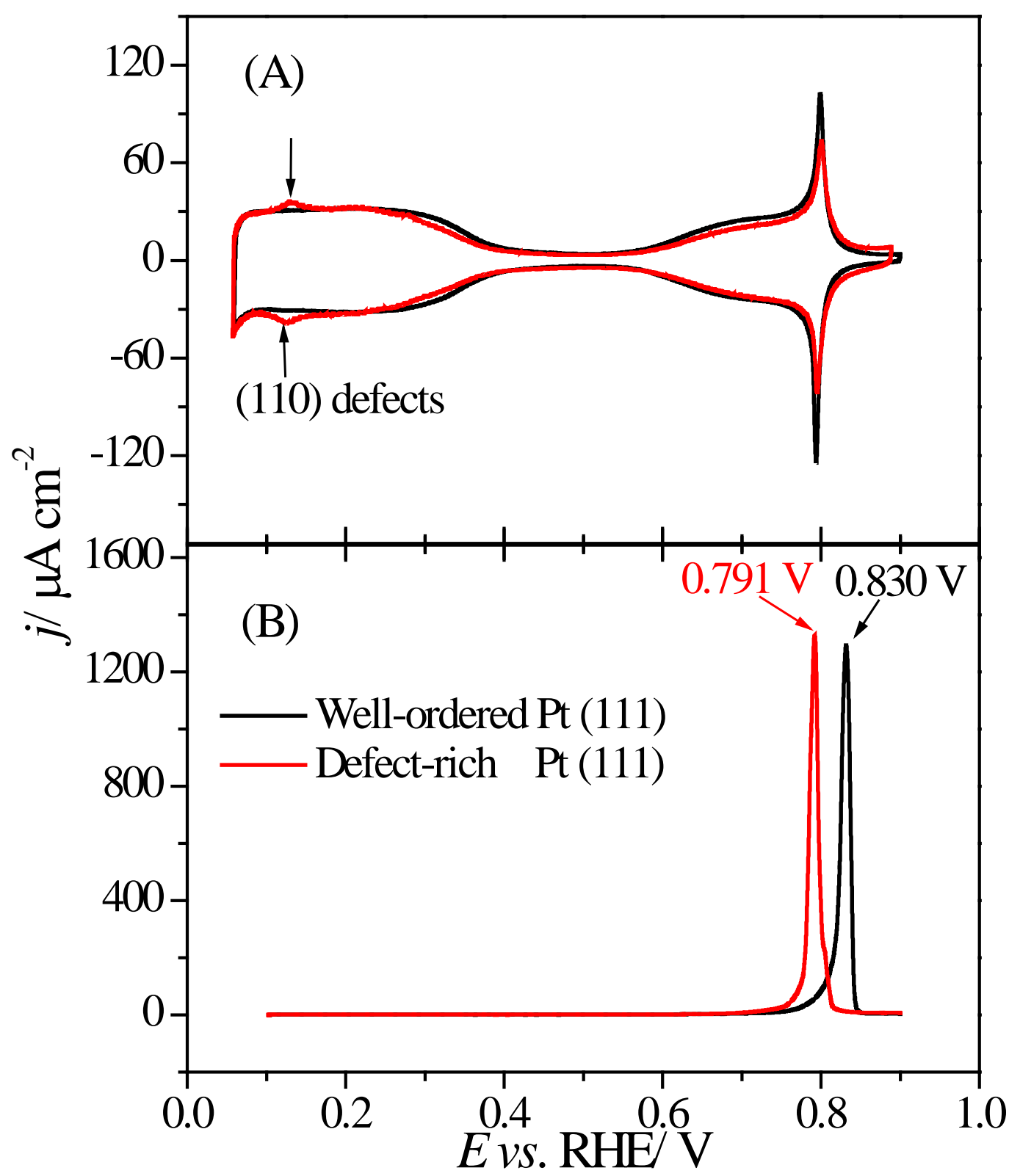

Figure 4, Farias et al. 


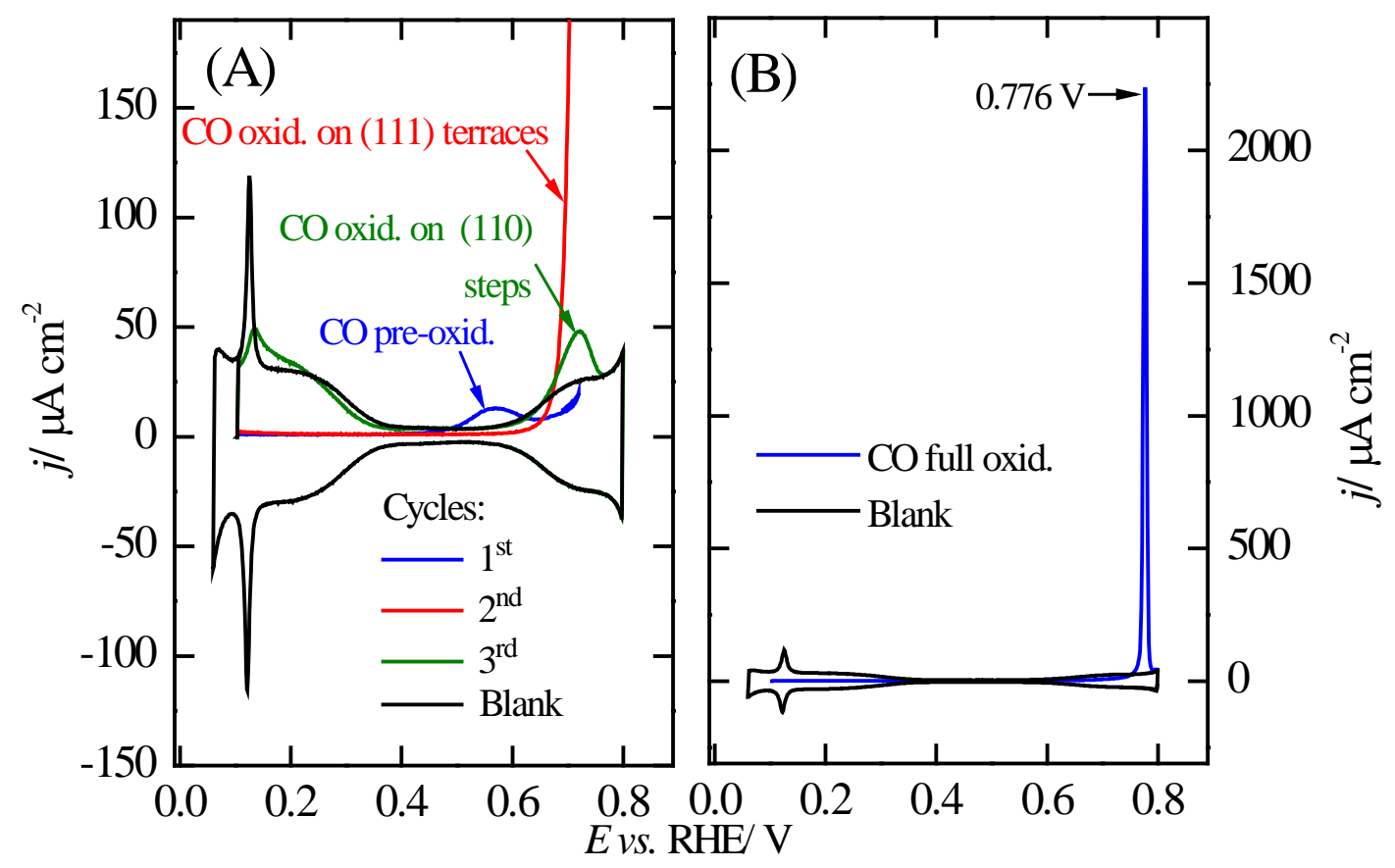

Figure 5, Farias et al. 

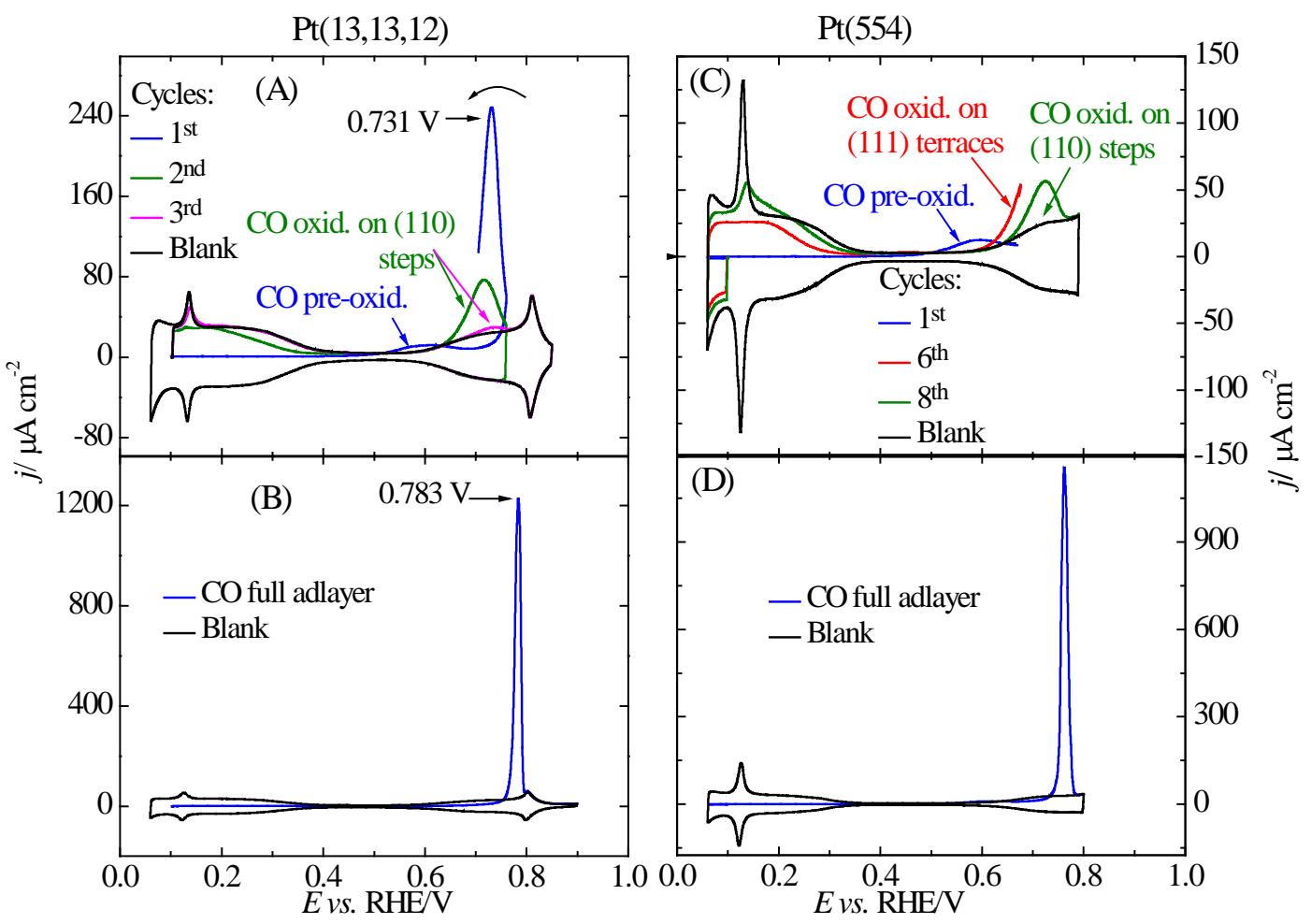

Figure 6, Farias et al. 


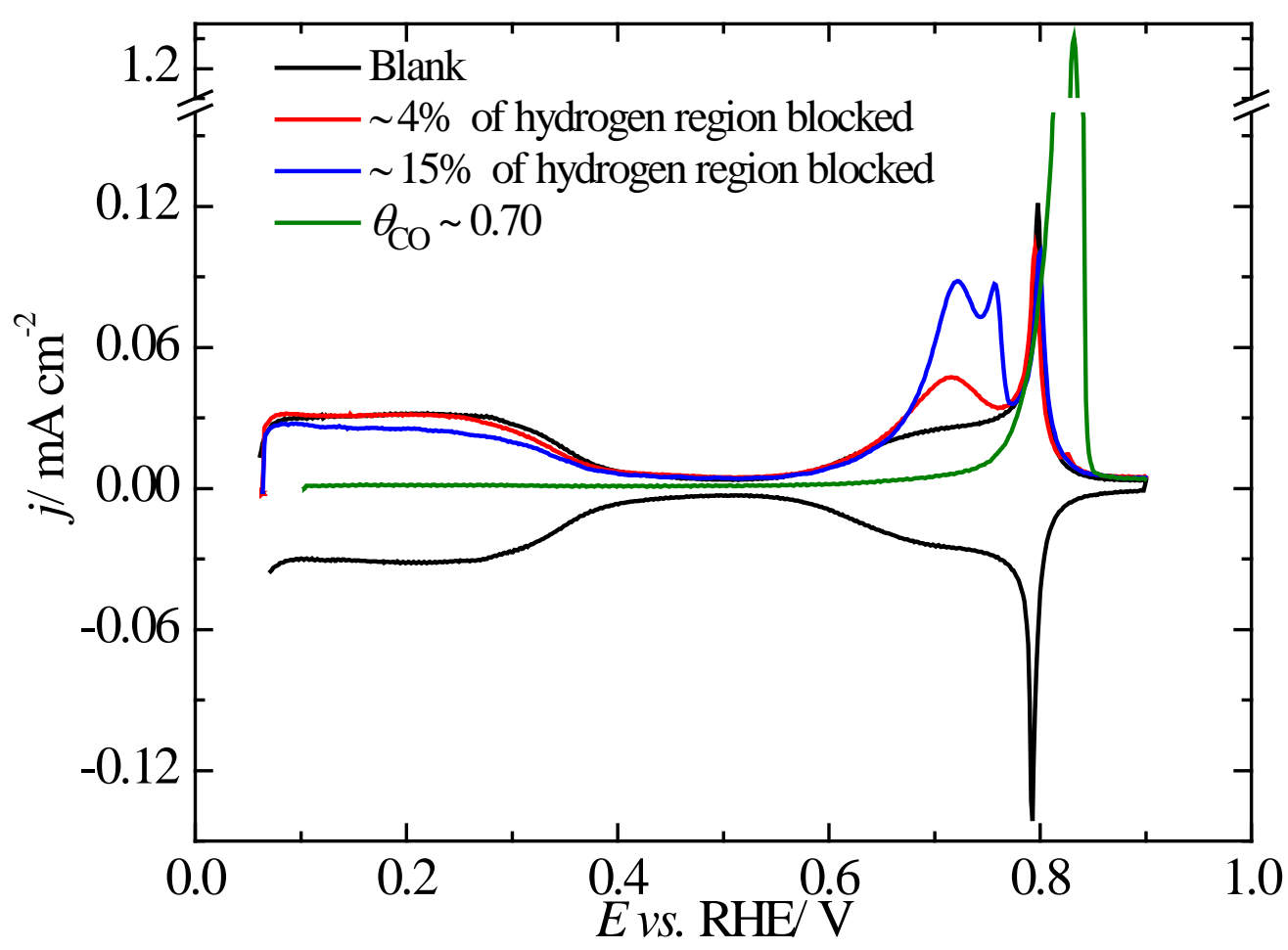

Figure 7, Farias et al. 


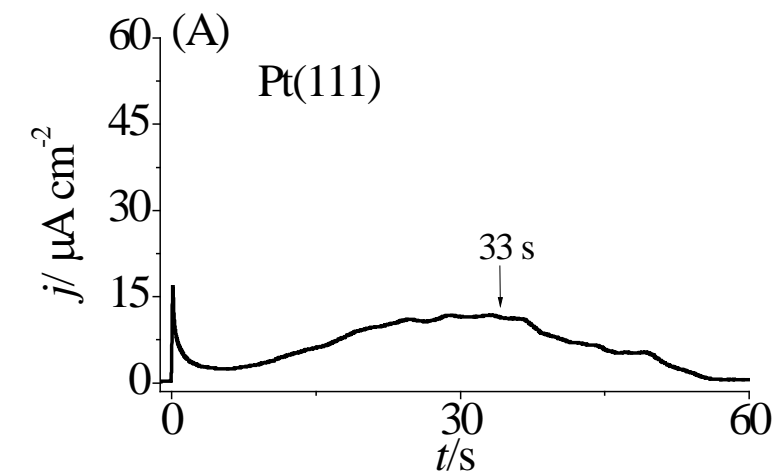

(B)

Pt(111)

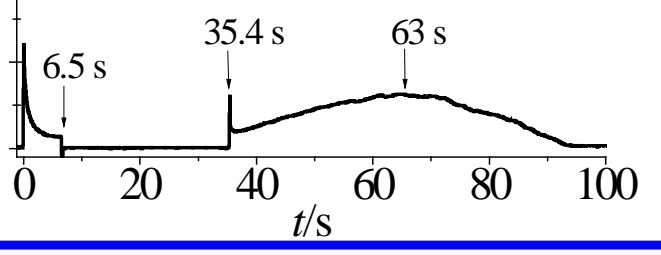

(C)

(D)
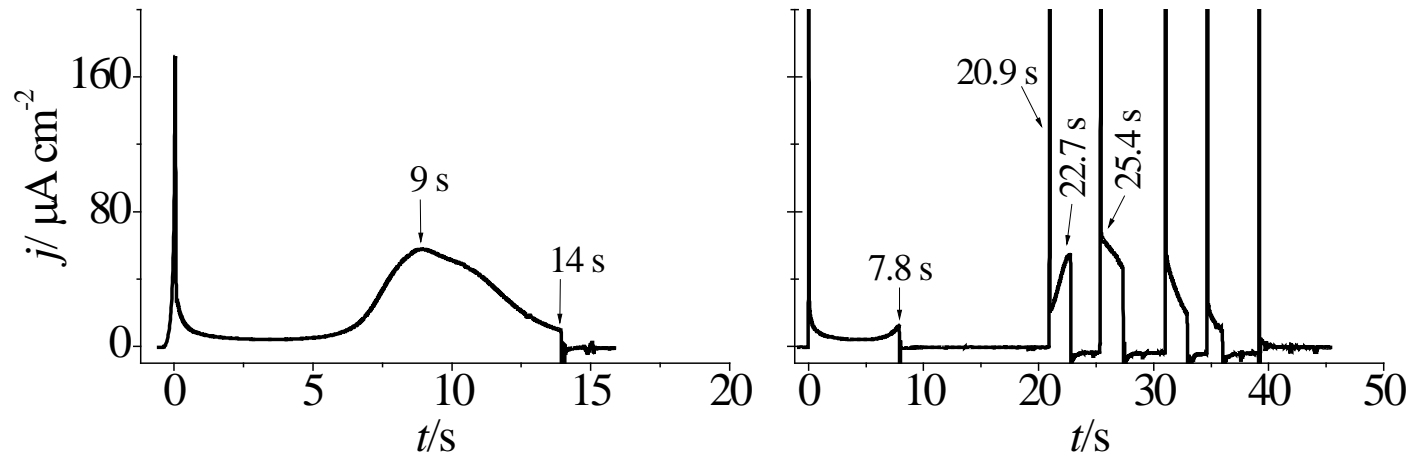

Figure 8, Farias et al. 


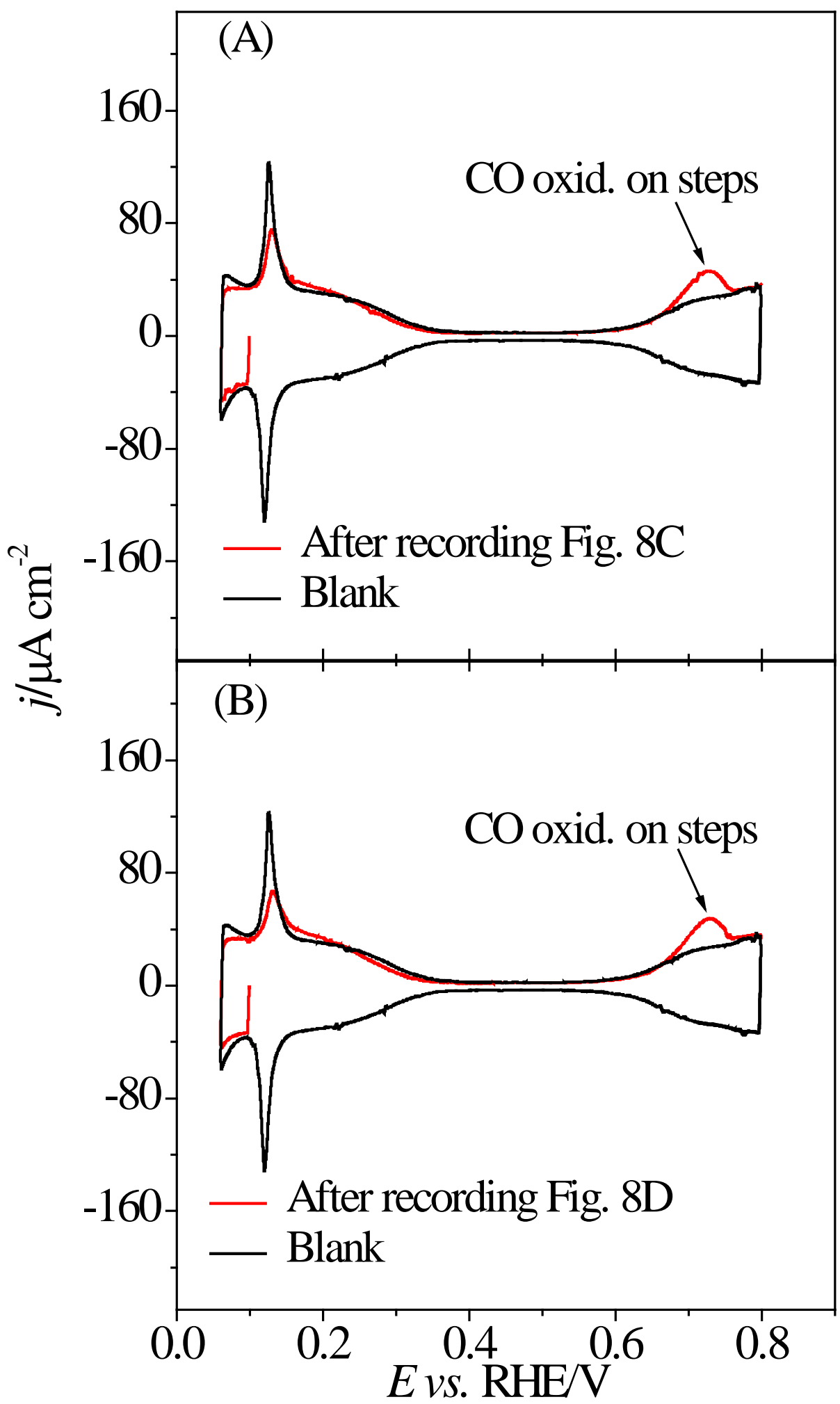

Figure 9, Farias et al. 
_ Full CO coverage

_ CO only (110) steps occupied

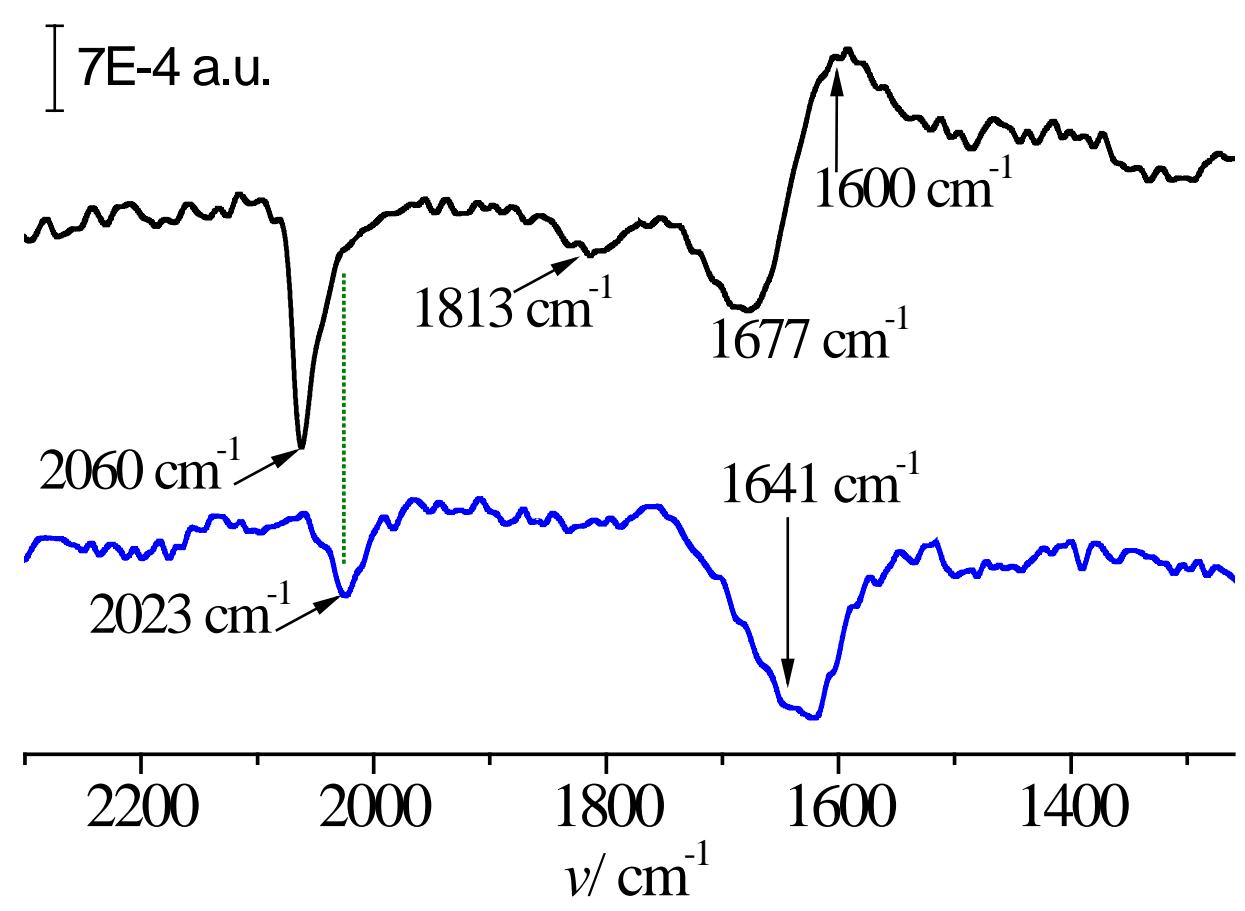

Figure 10, Farias et al. 